PHYSICS REVIEW

\author{
S. Mittal \\ Z. Wu \\ J. Neelavalli \\ E.M. Haacke
}

\section{Susceptibility-Weighted Imaging: Technical Aspects and Clinical Applications, Part 2}

SUMMARY: Susceptibility-weighted imaging (SWI) has continued to develop into a powerful clinical tool to visualize venous structures and iron in the brain and to study diverse pathologic conditions. SWI offers a unique contrast, different from spin attenuation, T1, T2, and T2* (see Susceptibility-Weighted Imaging: Technical Aspects and Clinical Applications, Part 1). In this clinical review (Part 2), we present a variety of neurovascular and neurodegenerative disease applications for SWI, covering trauma, stroke, cerebral amyloid angiopathy, venous anomalies, multiple sclerosis, and tumors. We conclude that SWI often offers complementary information valuable in the diagnosis and potential treatment of patients with neurologic disorders.
S usceptibility-weighted imaging (SWI) is a fully velocitycompensated high-resolution 3D gradient-echo sequence that uses magnitude and filtered-phase information, both separately and in combination with each other, to create new sources of contrast. With the advent of parallel imaging and the greater availability of clinical $3 \mathrm{~T}$ MR images, it is now possible to image the entire brain with SWI in roughly 4 minutes. SWI has been found to provide additional clinically useful information that is often complementary to conventional MR imaging sequences used in the evaluation of various neurologic disorders, including traumatic brain injury (TBI), coagulopathic or other hemorrhagic disorders, vascular malformations, cerebral infarction, neoplasms, and neurodegenerative disorders associated with intracranial calcification or iron deposition. As neuroradiologists become more aware of these various applications and as advances in software technology permit easier acquisition and better interpretation, SWI will likely be incorporated into the routine diagnostic imaging evaluation. The technical concepts of SWI were outlined in Part 1 of this 2-part review and would be valuable reading as background to this article, especially the discussion about magnitude, SWI filtered phase, SWI processed data, and contrast available in SWI. ${ }^{1}$ The following sections discuss different clinical applications of SWI predominantly in adults. An excellent clinical review by Tong et $\mathrm{al}^{2}$ in the American Journal of Neuroradiology already covers many SWI applications in children.

\section{TBI: Diffuse Axonal Injury}

TBI is a major cause of morbidity, mortality, disability, and lost years of productive life throughout the world. ${ }^{3} \mathrm{CT}$ remains the primary imaging technique for the initial evaluation of patients who have sustained head trauma because it effec-

From the Departments of Neurosurgery (S.M.), Biomedical Engineering (J.N., E.M.H.), and Radiology (E.M.H.) and the Karmanos Cancer Institute (S.M., E.M.H.), Wayne State University, Detroit, Mich; School of Biomedical Engineering (Z.W.), McMaster University, Hamilton, Ontario, Canada; and MRI Institute for Biomedical Research (J.N., E.M.H.), Detroit, Mich.

This work was supported by the National Institutes of Health Grant NHLBI R01 62983-04 and a grant from Siemens Medical Systems.

Please address correspondence to E. Mark Haacke, PhD, Wayne State University, MR Research Facility, Department of Radiology, HUH-MR Research G030/Radiology, 3990 John R Rd, Detroit, Ml 48201; e-mail: nmrimaging@aol.com

Indicates open access to non-subscribers at www.ajnr.org

DOI 10.3174/ajnr.A1461 tively allows detection of intracranial hemorrhages that require acute neurosurgical intervention. In recent years, however, MR imaging has been gaining popularity as an adjunctive imaging method in patients with TBI because it permits more precise identification and localization of smaller hemorrhages and can provide useful information regarding mechanisms of injury and potential clinical outcome. SWI is particularly helpful for the evaluation of diffuse axonal injury (DAI), often associated with punctate hemorrhages in the deep subcortical white matter, which are not routinely visible on CT or conventional MR imaging sequences. SWI exploits the magnetic susceptibility differences between tissues, resulting in phase differences between regions containing paramagnetic deoxygenated blood products (deoxyhemoglobin, intracellular methemoglobin, and hemosiderin) and surrounding tissue. The signal-intensity cancellation in the magnitude images and the additional suppression from the SWI filtered-phase images produce a hypointense signal in areas of acute and early subacute hemorrhage.

Studies by Tong et $\mathrm{al}^{4,5}$ and Babikian et $\mathrm{al}^{6}$ have shown that SWI is 3-6 times more sensitive than conventional $\mathrm{T} 2{ }^{*}$ weighted gradient-echo $\left(\mathrm{T} 2{ }^{\star} \mathrm{GE}\right)$ sequences in detecting the size, number, volume, and distribution of hemorrhagic lesions in DAI. Figure 1 shows a woman who was involved in a motor vehicle crash and sustained severe head injuries resulting in a coma. A nonenhanced CT scan showed suspicious low attenuations in the damaged areas. As expected, the cerebral lesions were more clearly visualized on T1- and T2-weighted images. SWI was even more strikingly sensitive in detecting the abnormalities and showed a much greater number and larger size of lesions compared with other conventional sequences. Furthermore, the shape of the lesions was more clearly delineated in the left cerebellar hemisphere, indicating the presence of shearing, often considered a cause of DAI.

Although larger hemorrhages are visible on CT and standard diagnostic MR imaging sequences, Tong et $\mathrm{al}^{5}$ have shown that numerous small hemorrhagic shearing lesions are only visible by using SWI. This finding is in agreement with ours. Furthermore, Babikian et $\mathrm{al}^{6}$ showed that the number and volume of SWI hemorrhagic lesions appear to correlate with specific neuropsychological deficits. The studies of Tong et $\mathrm{al}^{4,5}$ and Babikian et $\mathrm{al}^{6}$ also revealed that most patients had lesions in the frontal white matter or parietotemporal-occipital gray or white matter, whereas the thalamus, brainstem, cerebellum, and basal ganglia were less frequently affected. However, from our recent cases of patients 

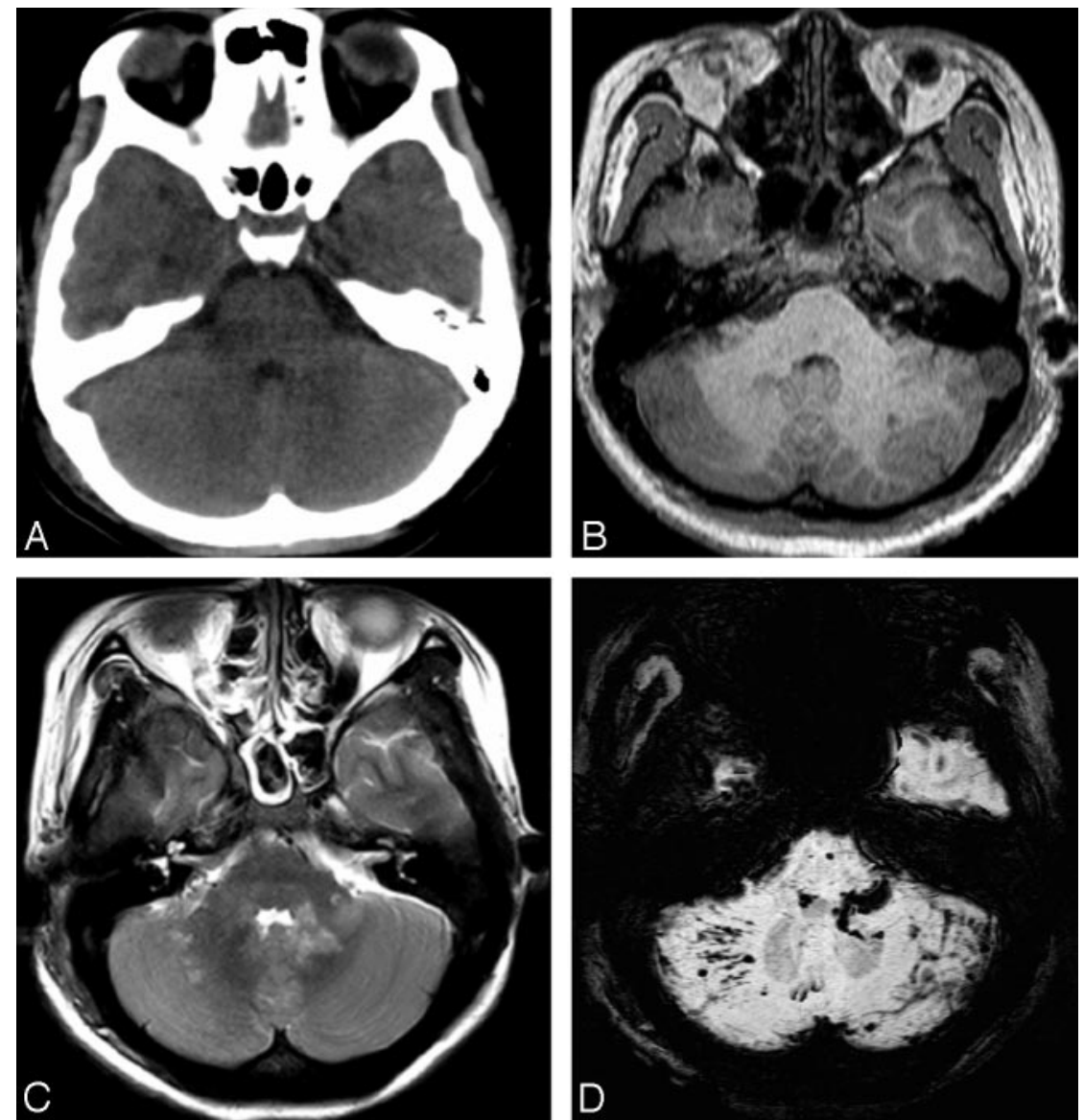

Fig 1. A 38-year-old woman who sustained severe TBI following a motor vehicle crash and lapsed into a coma. CT and MR images were obtained on the second day of hospitalization. $A$, Nonenhanced CT scan shows suspicious low attenuations in the pons and bilateral brachium pontis. $B$, T1-weighted image shows similar hypointense lesions in the pons, right brachium pontis, and right hemisphere of the cerebellum. $C$, T2-weighted image identifies multiple hyperintense lesions in the pons, bilateral brachium pontis, and right cerebellar hemisphere. $D$, SWI demonstrates multiple hypointense lesions in the left brachium pontis and both hemispheres of the cerebellum. There are more lesions shown on SWI, and they are also larger than those shown on CT and conventional MR imaging sequences. The shape of hemorrhagic lesions in the right cerebellar hemisphere reflects damage caused by shearing effect, indicating diffuse axonal injury. Courtesy of Dr Lei Jing, Tianjing Huanhu Hospital, Tianjing, China.
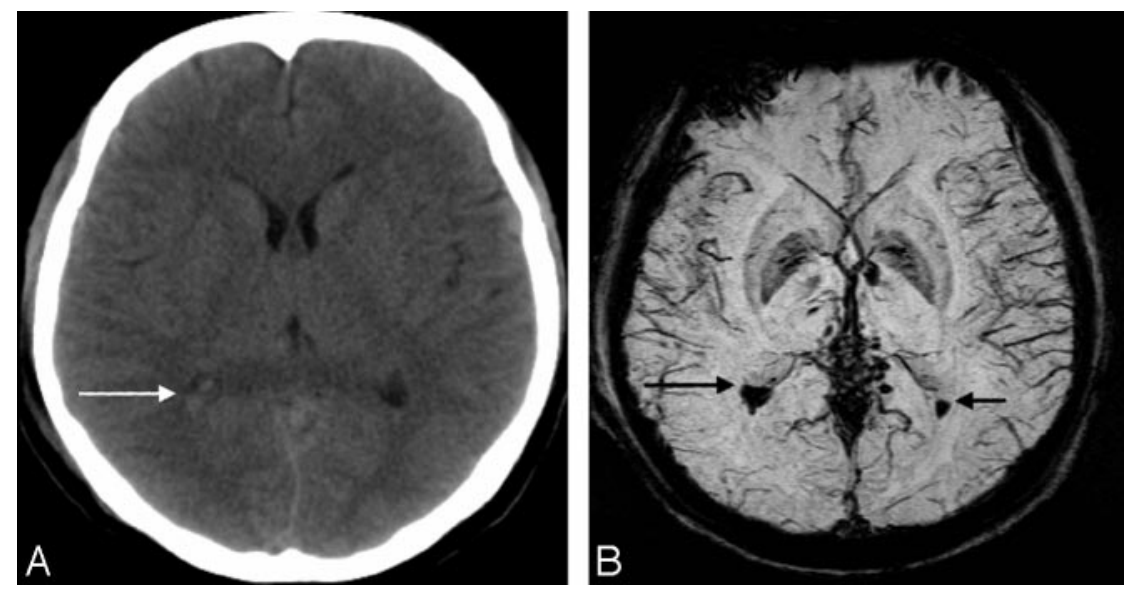

Fig 2. A, CT scan shows slightly higher attenuation (white arrow) in the posterior horn of right lateral ventricle. $B$, The SWI data clearly show hemorrhage (black arrows) inside both posterio horns of the lateral ventricle. Courtesy of Dr Lei Jing, Tianjing Huanhu Hospital, Tianjing, China.

with head injury, we have found that SWI is especially helpful in detecting traumatic lesions in the brainstem, whereas other MR imaging sequences have failed to demonstrate any such structural abnormalities.

The involvement of the brainstem in TBI is a very important predictor of long-term outcome. ${ }^{7}$ We have found that
SWI is not only sensitive in detecting hemorrhages occurring in the brain parenchyma but, in some cases, also shows intraventricular hemorrhage and subarachnoid hemorrhage, sometimes even better than CT. In Fig 2, CT failed to detect an intraventricular hemorrhage. SWI was performed on the same day and clearly showed hemorrhagic deposition in the poste- 

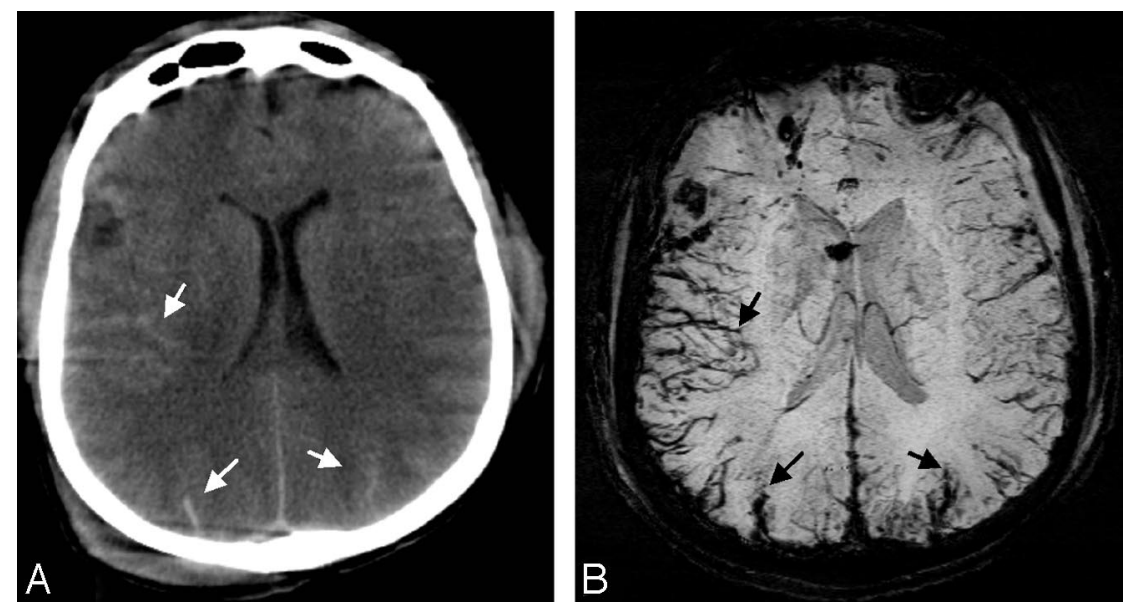

Fig 3. $A$, CT scan shows high-attenuation signals within the sulci of the cerebrum (white arrows), indicating traumatic subarachnoid hemorrhage. $B$, SWI clearly shows hemorrhage within the sulci of the cerebrum (black arrows), in agreement with the results of CT, but with a sharper contrast. Courtesy of Dr Lei Jing, Tianjing Huanhu Hospital, Tianjing, China.

rior horns of the lateral ventricles. The radiologist retrospectively confirmed the presence of a subtle high-attenuation signal intensity in the occipital horn of the right lateral ventricle on the initial CT scan. SWI was clearly helpful in making clinical decisions in this patient.

Another case of nonaneurysmal subarachnoid hemorrhage is shown in Fig 3. CT showed high attenuation along the sulci of the brain consistent with traumatic subarachnoid hemorrhage. SWI permitted visualization of the hemorrhage within the sulci with a sharper contrast than that on CT. For individuals not very experienced in reading SWI, this signal intensity change might be mistaken for veins. In this case, we have the impression that veins are shorter and do not follow the shape of the gyri perfectly. Further studies comparing subarachnoid hemorrhages in CT and SWI are needed to gain more experience in this area.

These findings in patients with TBI suggest that advanced neuroimaging techniques, including SWI, could play a very meaningful role in establishing the degree of injury more accurately, providing valuable prognostic information, and guiding the management and rehabilitation of patients with head injury. ${ }^{8}$

\section{Stroke}

Cerebrovascular ischemia from thromboembolism or arteriosclerotic stenosis leads to acute infarct with or without hemorrhage. In recent years, diffusion-weighted imaging (DWI), perfusion-weighted imaging (PWI), and MR angiography (MRA) have been incorporated in the initial assessment of acute stroke and have helped to direct further treatment. ${ }^{9}$ SWI is exquisitely sensitive in detecting hemorrhage and thus allows facile visualization of the hemorrhagic region. ${ }^{10-12}$ Thromboembolism can also change susceptibility by decreasing arterial flow, thus increasing the amount of deoxyhemoglobin, and may additionally cause increased pooling of deoxygenated blood.

Although DWI is a powerful method for detecting acute cerebral ischemia, SWI may be used as an adjunct to localize the affected vascular territory further. SWI, as a complementary sequence, can provide additional information by the following: 1) detecting a hemorrhagic component within the re- gion of infarction, further helping to distinguish ischemic and hemorrhagic stroke; 2) demonstrating areas of hypoperfusion and directing the necessity of PWI; 3) detecting acute thromboemboli that occlude arteries; and 4) predicting the probability of potential hemorrhagic transformation before thrombolytic treatment by counting the number of microbleeds and early detection of hemorrhagic complication after intra-arterial thrombolysis.

Studies have shown that SWI is more sensitive in detecting hemorrhage inside acute infarct lesions than CT and 2D gradient recalled-echo (2D-GRE) T2*-weighted imaging. ${ }^{13} \mathrm{SWI}$ is so sensitive to such a small amount of hemorrhage that even conventional long-TE GE images can fail to show the remnant hemorrhage after stroke, as shown in Fig 4.

SWI also shows prominent hypointense signals in the draining veins within areas of impaired perfusion. Uncoupling between oxygen supply and demand in hypoperfused tissue may cause a relative increase of deoxyhemoglobin levels and a decrease of oxyhemoglobin in the tissue capillaries and the draining veins. In Fig 5, a 59-year-old man with a history of atrial fibrillation presented with sudden-onset right hemiparesis and speech deficits. The MR imaging study at $1.5 \mathrm{~T}$ was performed 2 hours after the onset of symptoms and included conventional sequences, DWI, a dynamic-contrast perfusion study, and SWI. The mean transient time (MTT) map showed prolonged MTT in the left middle cerebral artery (MCA) territory. The areas with more prominent veins on SWI were in excellent agreement with the abnormal area on the MTT map. SWI can be used to anticipate the extent of impaired perfusion in acute cerebral ischemia without a contrast medium. It is possible to combine DWI and SWI to predict the "mismatch" of diffusion-perfusion. When SWI depicts prominently hypointense draining veins, a dynamic-contrast perfusion study should be used to evaluate vascular reserve such as relative cerebral blood flow ( $\mathrm{rCBF}$ ) and relative cerebral blood volume (rCBV).

In acute stroke, the demonstration of arterial clot with an accurate determination of its location helps to direct thrombolytic treatment. ${ }^{14}$ Fresh clots contain a high concentration of deoxyhemoglobin and appear hypointense on SWI. Clots located in the MCA have been studied by using susceptibility- 

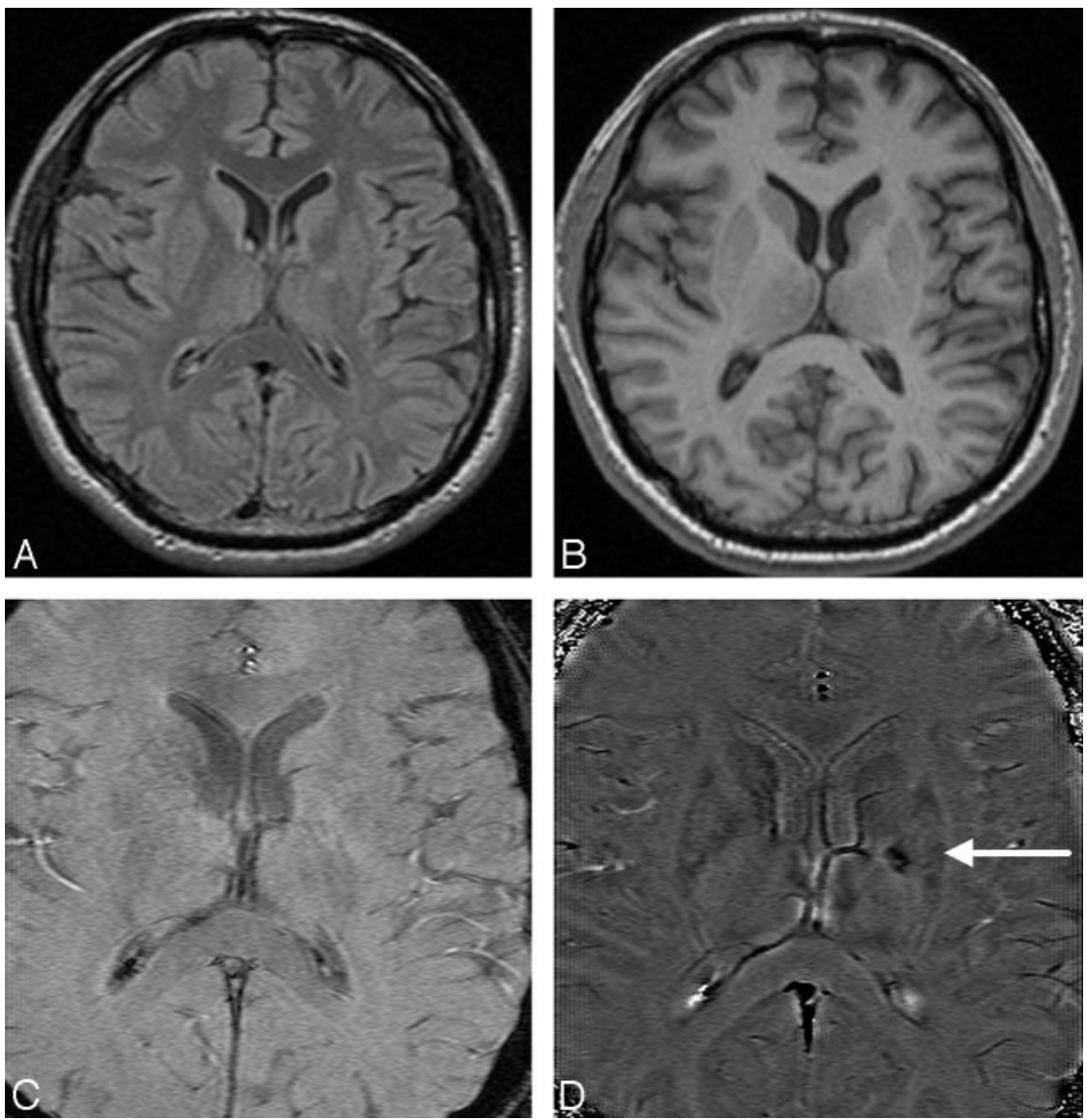

Fig 4. A patient who had sudden onset of aphasia and right paresthesias 5 years earlier and who partially recovered neurologic function after treatment of the acute stroke. $A$ and $B$, Findings of follow-up MR images are almost normal on the FLAIR $(A)$ and T1-weighted images $(B)$. $C$ and $D$, The original TE $=40$ ms magnitude image $(C)$ does not show any abnormality; however, the SWI phase image $(D)$ shows a small hemorrhagic lesion (white arrow) in the genu of the internal capsule and internal globus pallidus.

based perfusion MR imaging ${ }^{15}$ and GE imaging. ${ }^{16}$ As shown in Fig 5D, SWI shows hypointense signal intensity in the distal $\mathrm{M} 1$, representing the acute thromboembolus. For the proximal part of the cerebral arteries such as the M1 segment of the MCA, MRA can usually show the stenosis or occlusion very well; however, distal clots are not well visualized on MRA. However, Hermier et $\mathrm{al}^{17}$ have shown a case with a clot inside the left insular arterial branches of the MCA (M3) by using GE imaging. Likewise, SWI appears to complement MRA by being able to show clots in distal branches of cerebral arteries.

Hemorrhagic transformation is the most feared complication of thrombolytic treatment. Old microbleeds in patients with stroke have been studied in terms of their relationship to the potential for hemorrhagic transformation in the thrombolytic treatment of patients with acute stroke. ${ }^{18-21}$ Old microhemorrhages in a patient with stroke may reflect the vulnerability of the vascular system. ${ }^{21,22}$ If patients have a small number of microbleeds, thrombolytic treatment could be used safely, whereas having a large number of microbleeds may be a greater risk factor for hemorrhagic transformation. ${ }^{23}$ Several studies now suggest that SWI is the most sensitive method in detecting microbleeds, revealing up to 6 times more microbleeds than conventional GE methods. ${ }^{2,24}$ Figure 6 shows images from a 70-year-old man who underwent coronary artery stent placement and was placed on the antiplatelet agents aspirin and clopidogrel. Two months later, he presented with altered mental status. A follow-up CT scan showed intraparenchymal hemorrhage in the left posterior temporoparietal region. The patient underwent cerebral angiography and MRA, which demonstrated no evidence of arteriovenous malformation (AVM) or other pathologic vascular conditions. SWI, on the other hand, showed multiple old microbleeds in the cortical-subcortical interface, highly suggestive of cerebral amyloid angiopathy (CAA). Anticoagulation therapy was, therefore, immediately discontinued.

Intra-arterial thrombolytic therapy has become a common technique for the treatment of acute ischemic stroke in tertiary care centers. However, there is inherently a risk of hemorrhagic transformation following such treatment. Conventional imaging by using nonenhanced CT scans provides very limited information acutely following cerebral angiography. This is because the iodide-based contrast agent used during the angiography has high attenuation on CT. Therefore, it is very difficult to determine whether abnormal high attenuations on CT are related to acute hemorrhage or extravasation of dye from the artery. ${ }^{23,25} \mathrm{MR}$ imaging does not have this problem. A prospective blinded randomized trial has confirmed that $\mathrm{T} 2{ }^{*} \mathrm{GE}$ images can detect hemorrhagic transformation within 6 hours after intra-arterial thrombolysis. Because SWI is sensitive to deoxyhemoglobin, in theory, SWI could detect hemorrhagic transformation as early as several minutes after blood extravasation. Indeed, studies show that SWI can detect hemorrhagic transformation earlier than CT. ${ }^{17,19}$ 

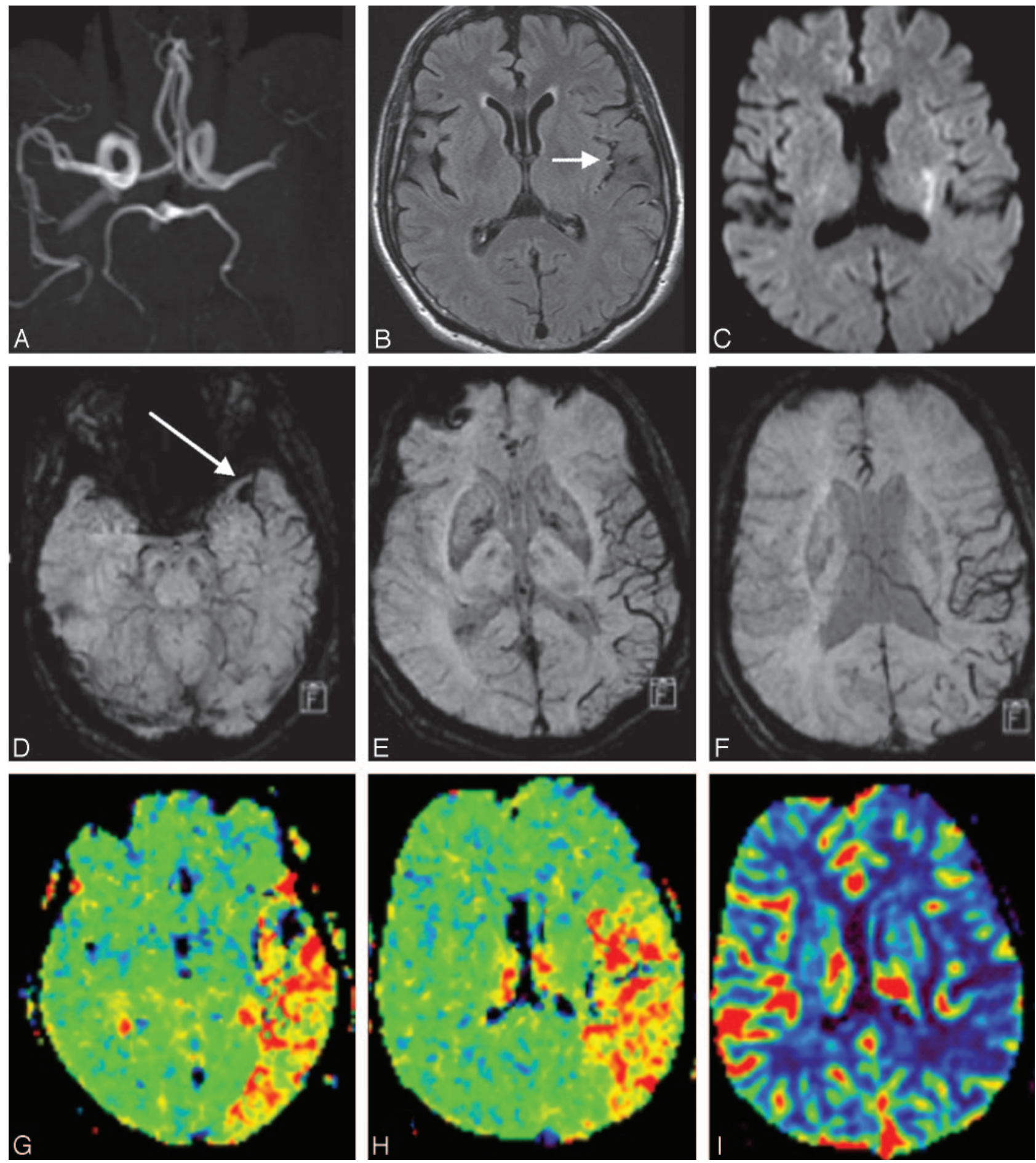

Fig 5. Cardioembolic stroke in the left MCA, 2 hours after onset. A, MRA shows lack of time-of-flight signal intensity in the distal M1 segment of the left MCA. B, FLAIR image displays intra-arterial signal intensity (arrow) of the left MCA branches along the lateral sulcus, indicating acute occlusion. C, DWI shows a small hyperintense lesion within the territory of the left MCA. D, SWI reveals localized hyposignal (arrow) in the left M1 segment, representing the acute thromboembolus itself. $E$ and $F$, SWI demonstrates prominently hypointense cortical veins within the left MCA territory, suggesting relatively increased deoxyhemoglobin in the draining veins within the acutely ischemic region. $G$ and $H$, The MTT map shows delayed transit times in the left MCA territory, matching SWI well. I, The rCBF is moderately reduced. Courtesy of Dr Masahiro Ida, Ebara Hospital, Tokyo, Japan.

CAA. Cerebrovascular disease is a major contributor to progressive cognitive decline in the elderly, accounting for perhaps as much as $30 \%-40 \%$ of dementia cases. Microvascular disease, which is not evident radiographically, almost certainly plays a role in the pathogenesis of dementia and also in Alzheimer disease. ${ }^{26} \mathrm{CAA}$ is a small-vessel disease characterized by the deposition of amyloid $\beta$ protein within the ce- rebral arterioles. CAA has a clear association with aging, dementia, Alzheimer disease, dementia pugilistica, postradiation necrosis, and spongiform encephalopathies. ${ }^{27}$ Currently, no in vivo imaging techniques exist to directly visualize or quantify the amyloid deposits. CAA can only be diagnosed histopathologically following biopsy or at postmortem examination. As the amyloid $\beta$ protein accumulates, the vessel walls 

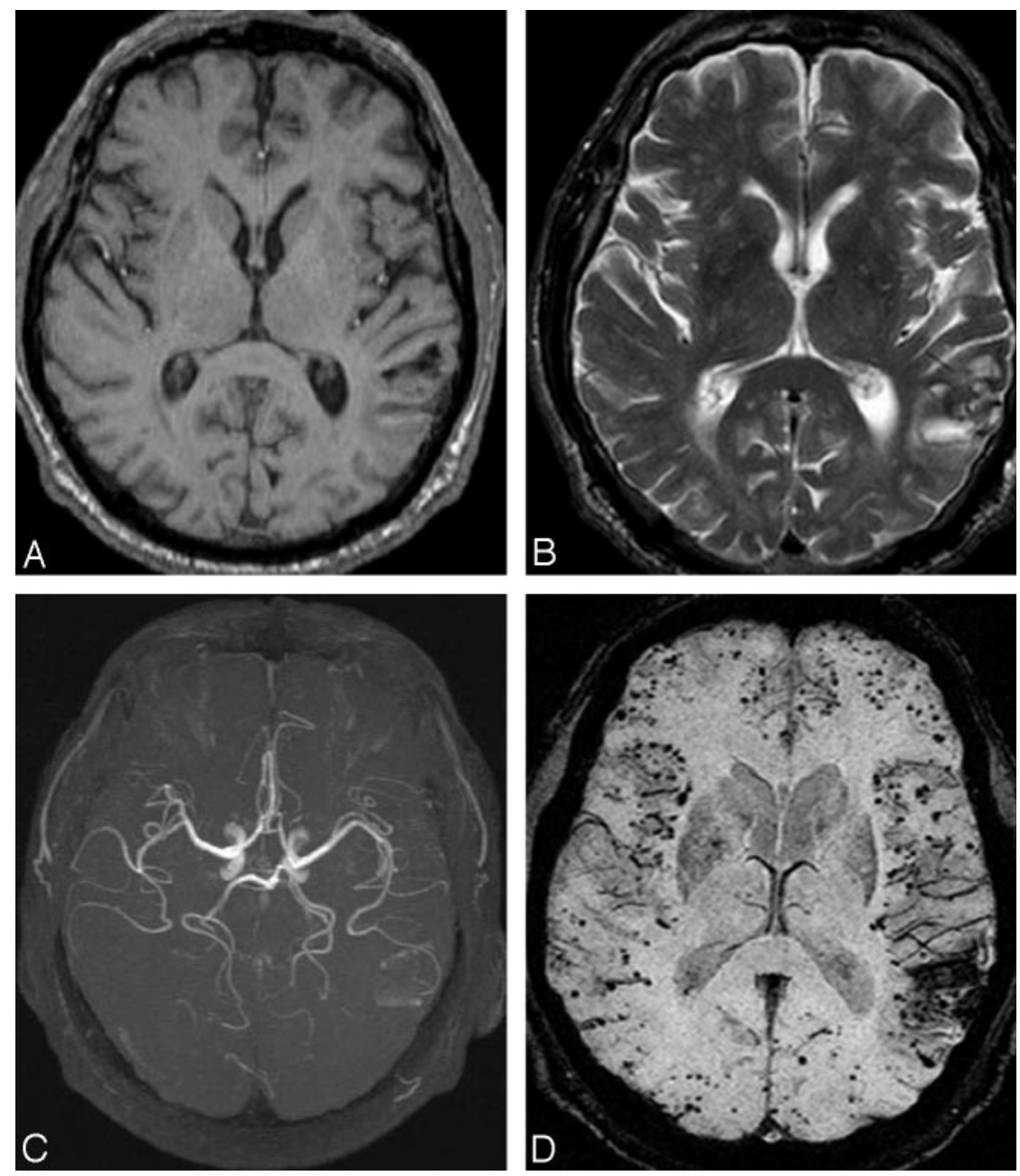

Fig 6. Multiple microbleeds in CAA. $A$ and $B$, T1-weighted $(A)$ and T2-weighted $(B)$ images do not reveal significant abnormalities except for the lesion in the left temporoparietal area. $C$, MRA shows normal brain vascular structure. $D$, SWI demonstrates, in addition to hemorrhage in the left temporoparietal region, multiple microbleeds distributed along the gray/white matter interface in the whole brain, strongly suggesting CAA.

become less elastic and more fragile. CAA typically results in microhemorrhages in and around the arteriole vessel wall. ${ }^{28}$ Lobar microbleeds are related to CAA (usually involving the cortex and subcortical white matter within the frontal and parietal lobes), whereas microhemorrhages in a deep or infratentorial location typically result from hypertensive or atherosclerotic microangiopathy. ${ }^{29}$ In addition to recurrent microbleeds, CAA can lead to intracranial lobar macrohemorrhages.

Conventional gradient-echo imaging is a well-established technique for detecting cerebral microhemorrhages. However, $25 \%$ of patients who were ultimately diagnosed with CAA did not have evidence of microbleeds on conventional T2 ${ }^{\star} \mathrm{GE}$ images. ${ }^{27}$ With its unique sensitivity to magnetic susceptibility effects, SWI is more detailed in identifying the microangiopathic changes seen in CAA. We recently evaluated a patient with suspected CAA by using SWI, ${ }^{27}$ and as seen in Fig 6 , SWI showed many microbleeds in the gray matter (and SWI also detected many more microhemorrhages than the conventional $\mathrm{T} 2{ }^{\star} \mathrm{GE}$ technique, not shown). Similar findings were reported by Larsen et al, ${ }^{30}$ who evaluated an 88 -year-old woman with Alzheimer disease and advanced dementia. Overall, SWI is a more sensitive diagnostic tool, which may permit more precise assessment of the natural history of CAA and response to therapy.
Lacunar Infarcts. Lacunar infarcts are attributed most commonly to deep penetrating arterial occlusion of the lenticulostriate arteries, anterior choroidal artery, paramedian branches of the basilar artery, and thalamoperforator branches of the posterior cerebral artery. Lacunar infarcts are seen relatively commonly, and it is thought that most of these infarcts are thrombotic manifestations of atheromatous disease. Lacunar infarcts may also be hemorrhagic in origin. SWI can detect small lacunar infarctions and shows promise for visualizing the extent of longstanding cerebrovascular disease. Another potential benefit of SWI may be the detection of distally located clots, which may be missed by MRA. ${ }^{10}$

Schellinger et $\mathrm{al}^{9}$ have proposed a multimodal stroke MR imaging protocol (including T2, fluid-attenuated inversion recovery [FLAIR], GRE T2*, DWI, PWI, and MRA), which may be the single diagnostic tool of choice in the initial assessment of patients with hyperacute ischemic or hemorrhagic stroke. It is our impression that substituting the conventional GRE T2*-weighted images with the SWI sequence as described in this review will improve the sensitivity of MR imaging in the detection of hemorrhage in an acute stroke. ${ }^{13}$ An example of ischemic stroke is shown in Fig 7, where a severe stenosis in the left carotid artery resulted in small lacunar infarcts. This modified combination of sequences can be suf- 

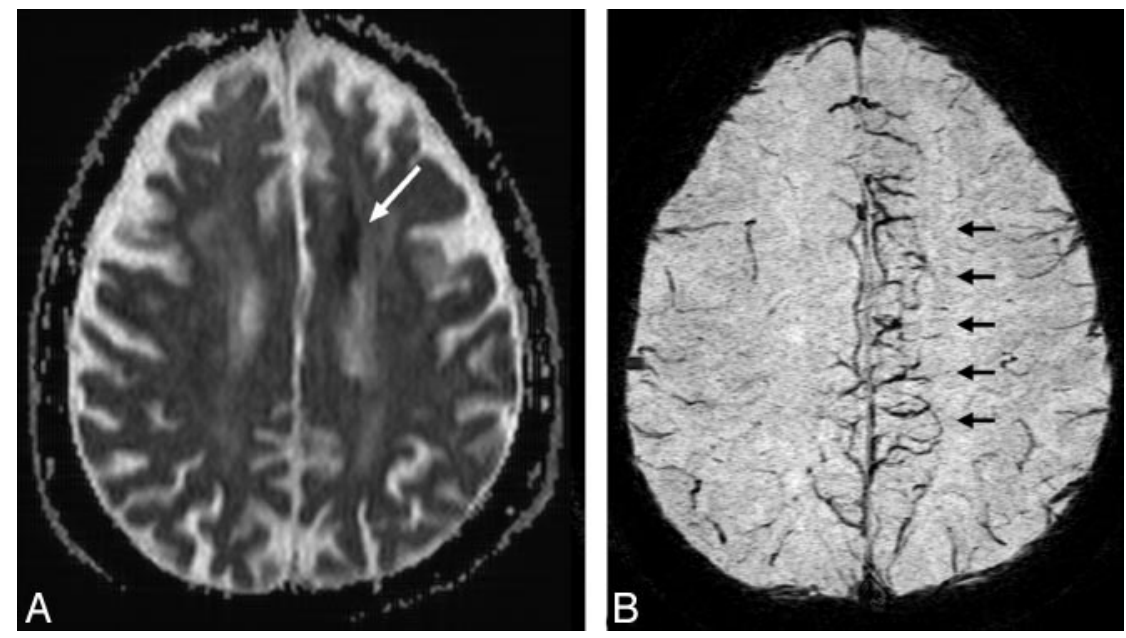

Fig 7. An acute stroke patient with severe stenosis of the left internal carotid artery. The apparent diffusion coefficient map $(A)$ shows an acute infarction as a low signal intensity (white arrow) together with old infarctions (which appear bright). The SWI data $(B)$ shows prominently hypointense cortical veins reflecting the reduced oxygenation levels in the left anterior cerebral artery territory (arrows). Courtesy of Jonathon Grynspan, McMaster University, Hamilton, Ontario, Canada.

ficient, if not superior to other techniques, in the rapid evaluation of patients with acute stroke for triage regarding thrombolytic therapy and may eliminate the need for CT in the initial evaluation of patients with suspected stroke.

Cerebral Autosomal Dominant Arteriopathy with Subcortical Infarcts and Leukoencephalopathy. Cerebral autosomal dominant arteriopathy with subcortical infarcts and leukoencephalopathy (CADASIL) is the most common form of hereditary stroke disorder and is thought to be caused by mutations of the notch3 gene on chromosome $19 .{ }^{31}$ The most common clinical manifestations are migraine headaches and transient ischemic attacks or strokes, which usually occur between 40 and 50 years of age. The underlying pathology of CADASIL is progressive degeneration of the smooth muscle cells in blood vessels. Lacunar infarcts appear to correlate with cognitive dysfunction in CADASIL. MR imaging shows hypointensities on T1-weighted images and hyperintensities on T2-weighted images. Multiple confluent white matter lesions of various sizes are characteristic and well-demonstrated on SWI (Fig 8).

\section{Neurodegenerative Disorders}

It is well documented that iron content in the brain increases with age, particularly in the basal ganglia, and that abnormal levels of iron in the central nervous system (CNS) are seen in various neurodegenerative diseases. ${ }^{32,33}$ In fact, increased iron deposition occurs in Parkinson disease, Huntington disease, Alzheimer disease, multiple sclerosis (MS), amyotrophic lateral sclerosis, Hallervorden-Spatz syndrome, and pantothenate kinase-associated neurodegeneration (PKAN). ${ }^{34-37}$ The ability to measure the amount of nonheme iron in the brain will facilitate a better understanding of disease progression and will also help in predicting the treatment outcome. Minerals such as calcium (diamagnetic) and iron (paramagnetic) exert different degrees of susceptibility effects, thereby enabling SWI to be a more sensitive means of detecting mineral deposits. SWI filtered-phase images have been shown to be useful for observing increased iron content in the brain. ${ }^{12,38}$ However, in any patient, it will be difficult to differentiate normal and pathologic mineralization. The iron difference be- tween gray matter and white matter can lead to excellent contrast in areas such as the motor cortex, other areas of gray matter, and even in the pons (Fig 9). We recently used SWI to determine the baseline phase behavior patterns due to iron content or mineralization in multiple brain regions. ${ }^{39}$ Further work is needed to determine the usefulness of SWI in differentiating normal and abnormal mineral deposition in the human brain.

Genetic and molecular-biologic studies of iron metabolism in the brain have suggested that at least in some neurodegenerative disorders, brain iron misregulation is an initial cause of neuronal death. Iron-induced oxidative stress is the most common path that leads to the death of neurons. ${ }^{40}$ If abnormal iron deposition is a primary cause of brain tissue injury, MR imaging can be used to detect the disease process in its early stages and may perhaps be helpful in monitoring the pharmacologic results because iron will be the therapeutic target. If iron deposition is just a secondary consequence or a standby phenomenon of the development of disease, then iron and perhaps SWI filtered-phase data can be used as a marker of disease severity. Recent studies by using T2 methods to estimate iron content have found the following: 1) There are lateral substantia nigra pars compacta abnormalities in early Parkinson disease ${ }^{41}$; 2) there is a significantly increased percentage of low-T2 voxels in the hippocampus, the ventral pallidum/nucleus basalis of Meynert, and the globus pallidus in Alzheimer disease ${ }^{42}$; and 3) four neurodegenerative diseases including PKAN, neuroferritinopathy, infantile neuroaxonal dystrophy, and aceruloplasminemia can be reliably differentiated according to their characteristic abnormal iron distributions in different nuclei. ${ }^{43}$ However, measuring the shortening of the $\mathrm{T} 2$ to calculate the iron distribution is empiric and cannot precisely calculate the iron concentration quantitatively. ${ }^{44}$

\section{MS}

MS is an acquired inflammatory disease affecting the brain and spinal cord. Demyelinating plaques are typical and result in episodes of neurologic deficit with recovery (relapses) and accumulation of sustained disability with the passage of time. Subcortical white matter lesions, irregular and confluent 

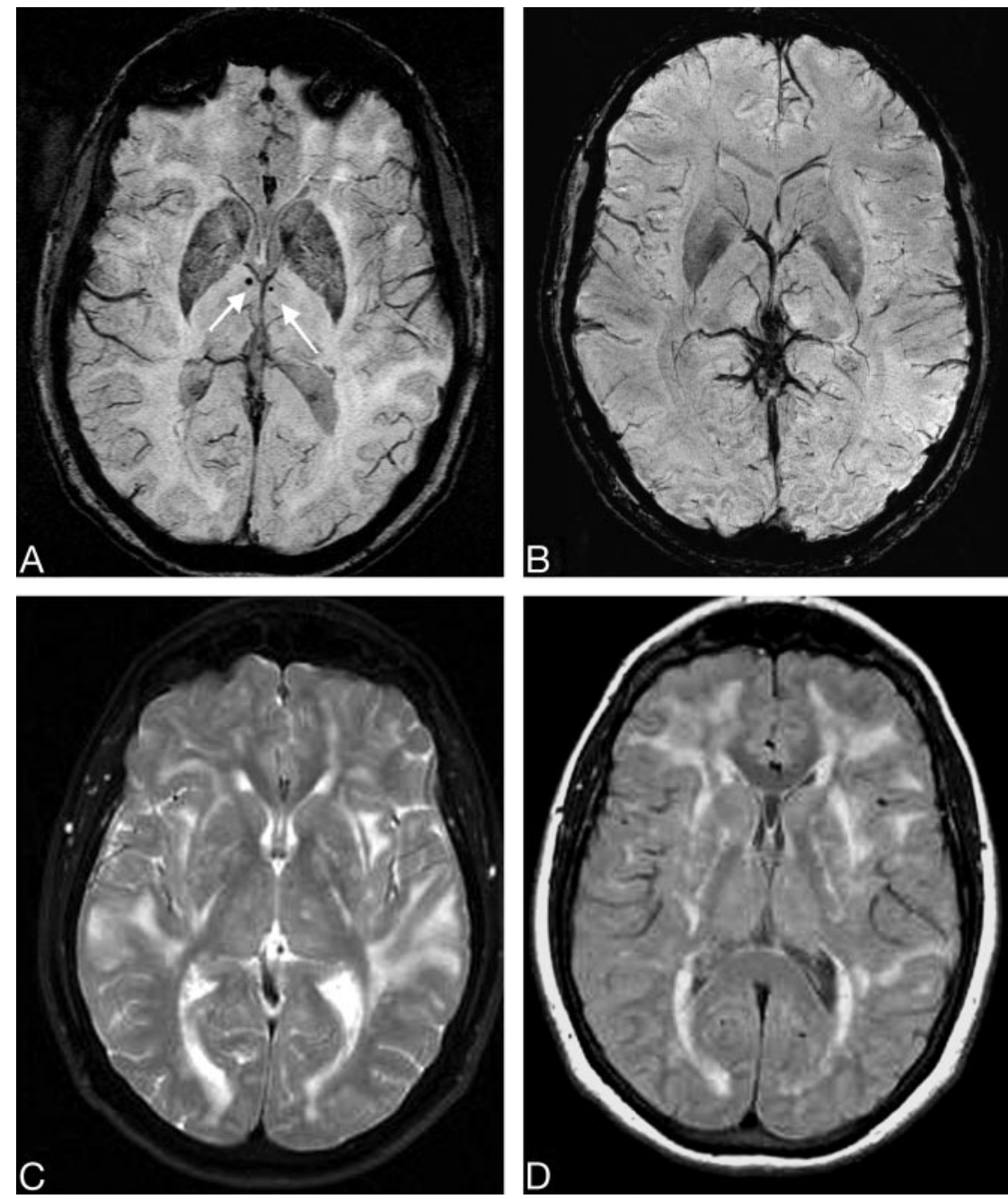

Fig 8. $A$, The SWI processed data for this 57-year-old patient with CADASIL shows 2 small bleeds in the anterior thalamus (arrows) and increased iron content in the basal ganglia. $B$ The SWI processed image for a healthy 53 -year-old volunteer shows significant iron only in the globus pallidus. $C$ and $D, T$ The T2 (C) and T1 (D) spin-echo images of the patient with CADASIL show diffuse white matter hyperintensities.
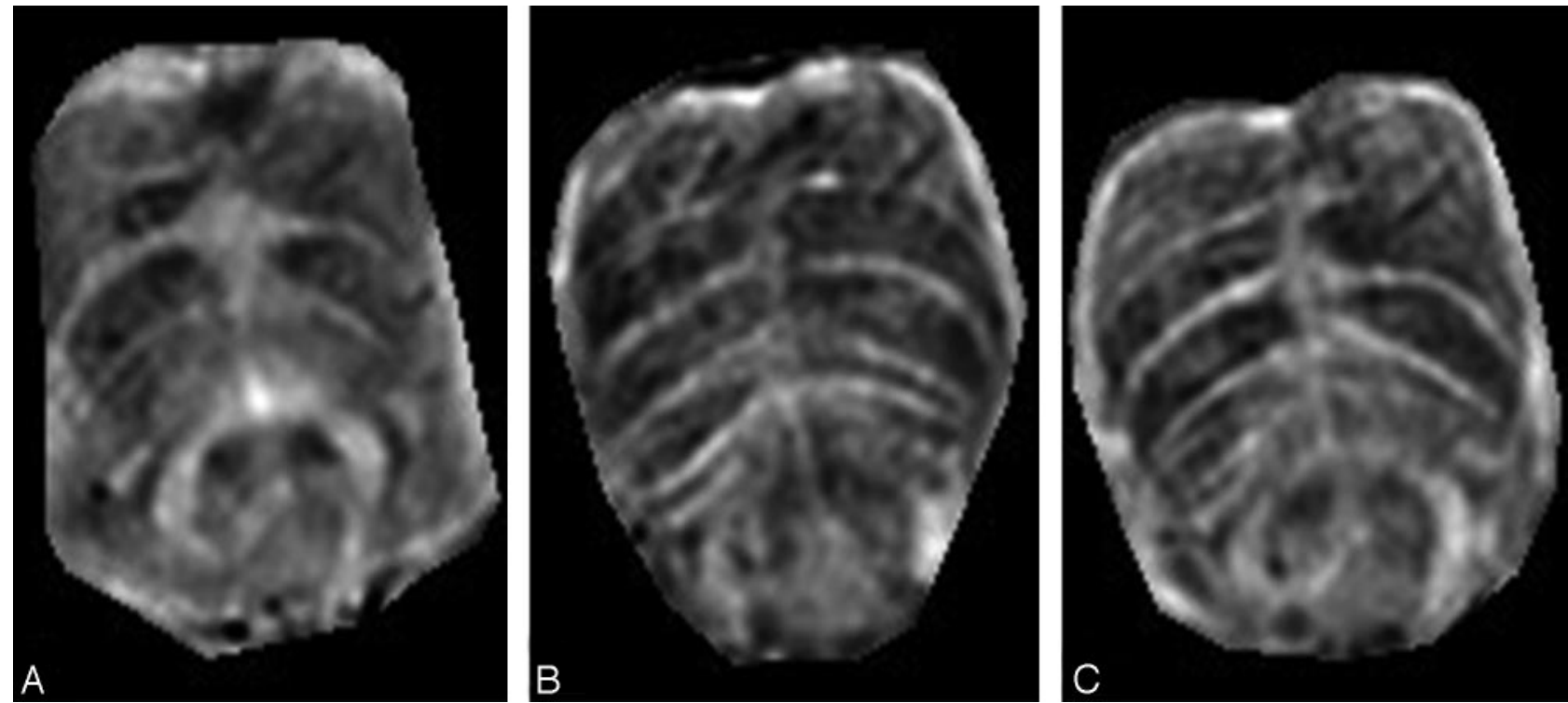

Fig 9. SWI data for 3 slices through the pons of a progressive MS patient with severe iron build up in various parts of the brain. 

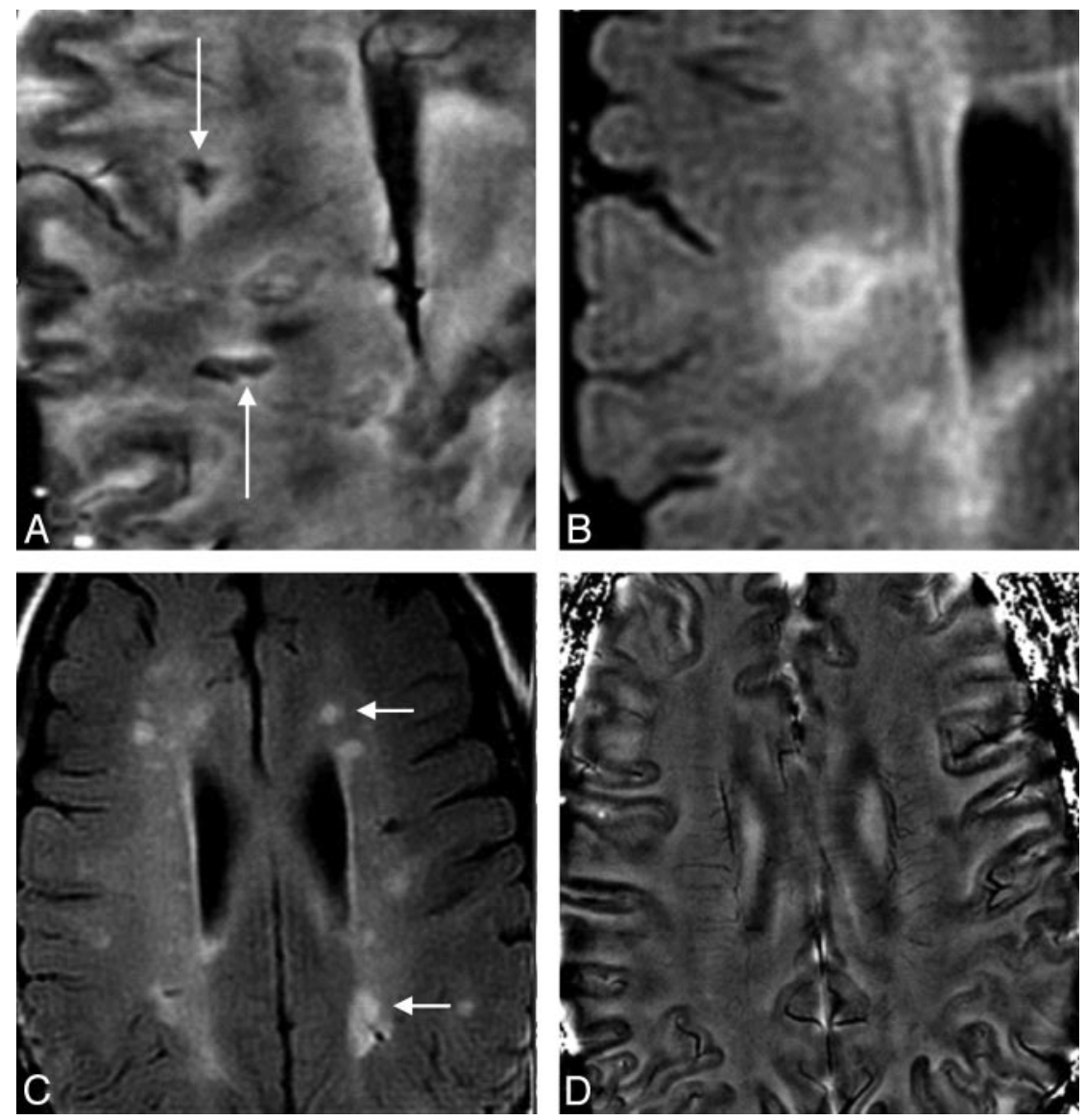

Fig 10. Different lesion distributions on SWI phase images and conventional MR images at 4T. $A$ and $B$, Two lesions (long arrows) shown on the SWI phase image (A) are barely seen on FLAIR $(B)$. C and $D$, On the other hand, lesions (short arrows) along the lateral ventricles shown on FLAIR $(C)$ are not obvious on the SWI phase image $(D)$.

periventricular lesions, or lesions in the corpus callosum are highly suggestive of MS, which is usually studied with FLAIR and contrast-enhanced T1 imaging. Although the sensitivity of MR imaging in depicting MS lesions in the brain is very high, ${ }^{45}$ its specificity is low.

One way to increase specificity is to have the ability to image details like lesion topology. ${ }^{46}$ The morphology of lesions is also important: Ovoid lesions and so-called Dawson fingers are commonly found in MS. ${ }^{46}$ The distribution and shapes of MS lesions have been well-depicted on MR images and histopathologic analyses. These studies have shown that the topography and morphology of the lesions relate to the origin of plaques around a perivenous inflammation. ${ }^{47}$ Moreover, autopsy studies have demonstrated that the typical periventricular distribution of MS lesions can be explained by their perivenous location. ${ }^{48,49}$ It has been suggested that abnormalities of the periventricular deep medullary veins could be important in diagnosing MS onset and progression. ${ }^{49} \mathrm{Tan}$ et al ${ }^{46}$ have used high-resolution MR venography (which can depict veins in the brain with a diameter as small as $1 \mathrm{~mm}$ ) to show the perivenular distribution of demyelinating lesions, confirming that the shape of MS plaques is determined by the location and orientation of veins. There were 95 lesions in total from the 17 patients with MS in the Tan et al study, ${ }^{46}$ which showed a vein running centrally in all except 1 subcortical lesion. The orientation and form of the MS lesions correlated well with the course of this vein, confirming their perivenous origin.

Similarly, Hammond et a ${ }^{50}$ studied 7 patients with MS and observed small phase-shifted plaques in the region of the deep medullary veins; this observation supports the contention that the disease progresses along the vasculature. They found that a subset of MS plaques showed contrast uptake only at the peripheral margin of the lesion in the phase images. This region may correspond to the zone of active inflammation, with an influx of iron-rich macrophages in the postgadolinium images of acute plaques, and may also contain globular structures of nonheme iron, as seen in histologic studies of older plaques. ${ }^{32}$ As such, chronic MS lesions may show evidence of iron deposition, which may be better detected by SWI than by conventional GRE. ${ }^{12}$ Thus SWI enables precise in vivo visualization of the venous architecture of the CNS and can help improve our understanding of the pathophysiology and typical periventricular distribution of MS lesions (Fig 10).

A comparison of the phase shift with gadolinium enhancement could potentially be used to differentiate past (chronic) from present (acute) inflammation, with the quantity of deposited blood products in chronic plaques possibly indicating the level of past activity. ${ }^{50}$ More recently, our group has completed a study of 27 patients with MS by using SWI filteredphase images. ${ }^{51}$ We found that there is a negative correlation of iron with T2 hyperintensity. The higher the iron content, 

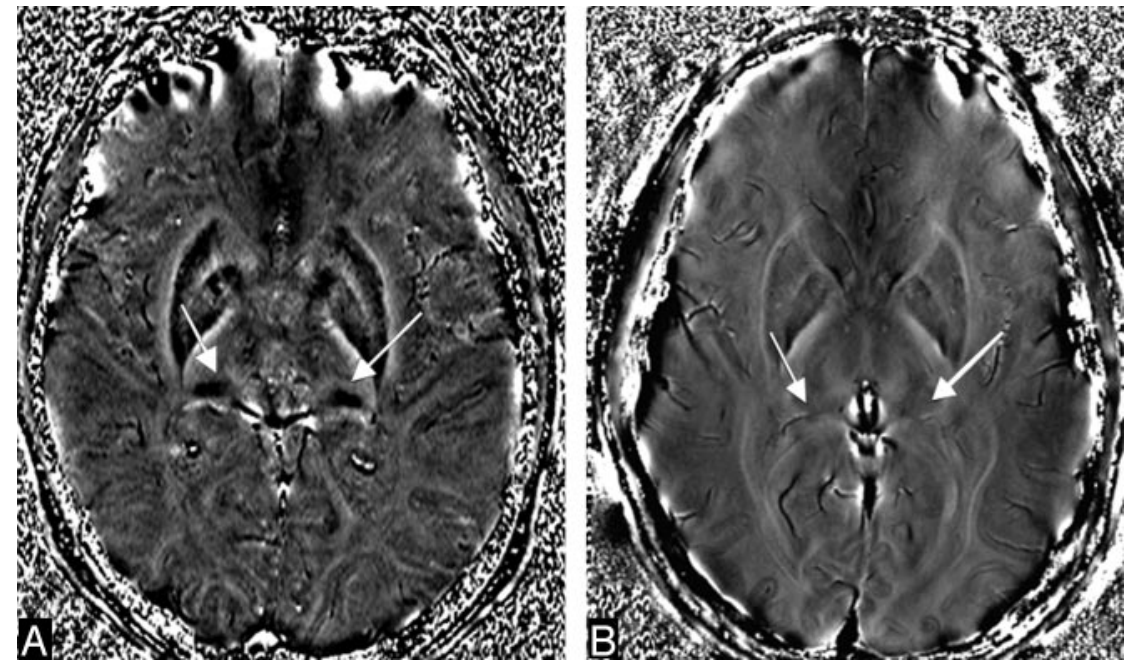

Fig 11. In a patient with MS, iron builds up not only in the basal ganglia but also in the pulvinar nucleus of the thalamus. $A$, The SWI image for the patient shows the areas of increased iron content, including the pulvinar. $B$, In the healthy age-matched volunteer, there is little iron deposition in the pulvinar nucleus.
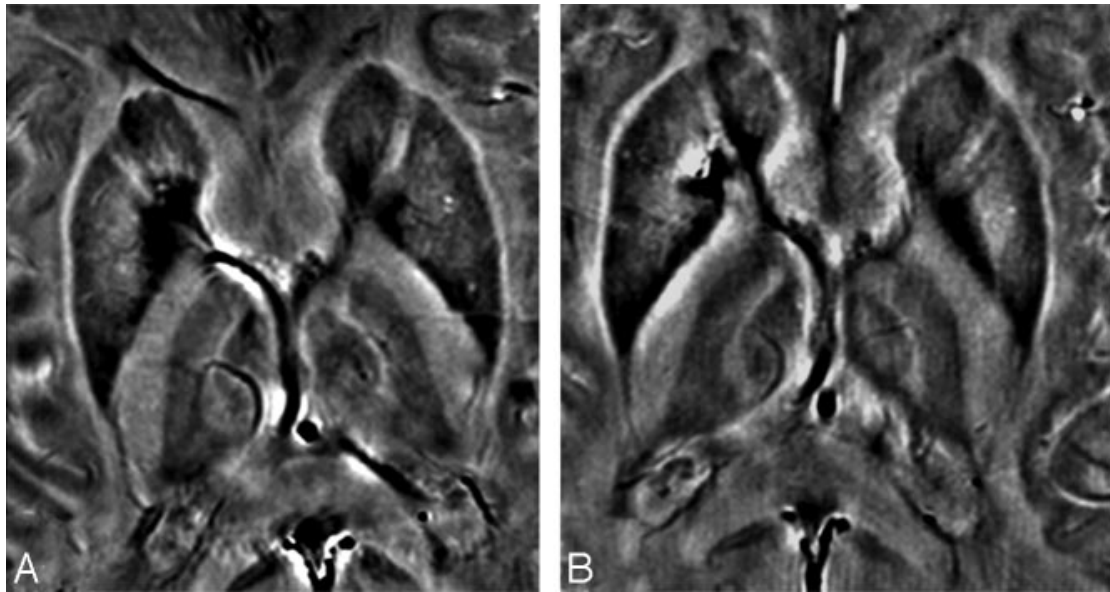

Fig 12. SWI data in a patient with progressive MS exquisitely demonstrate the location of iron deposition in the deep gray matter. The second scan (B) was acquired 1.5 years after the first scan $(A)$ and shows an increase in the iron content in the globus pallidus as demonstrated by the increased phase values and aliasing seen in $B$.

the less visible the lesions seem to be in T2-weighted images. This may suggest that significant damage to the tissue occurs following chronic inflammation, such that the tissue no longer shows signs of inflammation. We also noted that there were marked hypointense rings around some lesions, lesions with uniform iron content, and regions with central iron but no rings. On occasion, lesions were also seen in the gray matter as well. Overall, in that particular study, using SWI permitted a more accurate measurement of the extent of MS and increased the number of lesions detected by $50 \%$.

SWI filtered-phase images have been shown to be useful for observing increased iron content in the brain. ${ }^{12,38}$ Because the phase value is a linear function of iron concentration, the iron content can be quantitatively measured more easily than by using T2 methods. We recently determined the baseline phase values by measuring 75 people (older than 65 years of age) with normal iron content in many brain structures. ${ }^{39}$ The higher the iron content, the less visible the lesions seem to be in T2-weighted images. In patients with MS, very high iron deposition was seen in certain deep gray matter structures such as the pulvinar (Fig 11), caudate nucleus, putamen, and thalamus (Fig 12). In Fig 13, so much iron was deposited in the

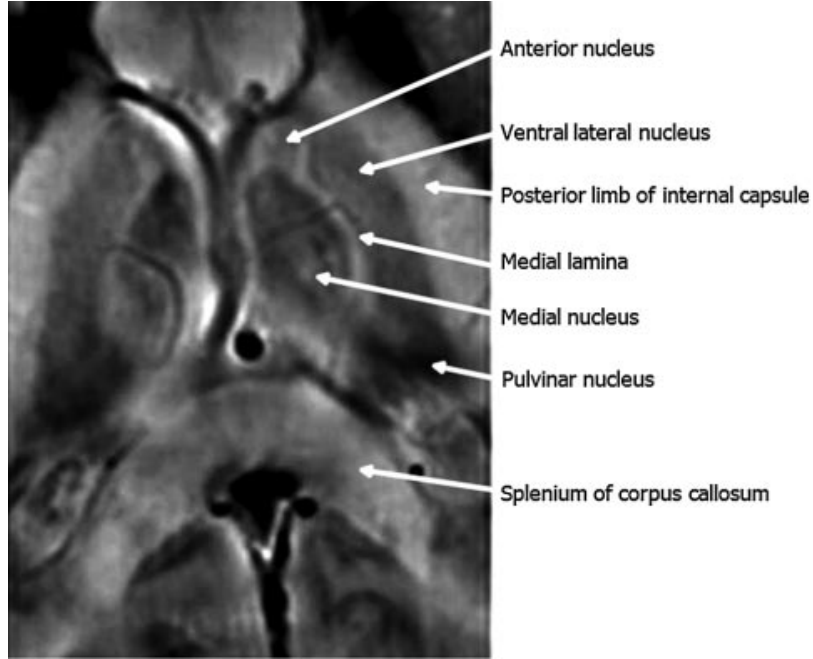

Fig 13. Detailed visualization of the thalamic structures by using SWI filtered-phase images in a patient with MS.

thalamus that the different nuclei within the thalamus were clearly visible. As mentioned above, iron is also seen in the ringlike structures around some MS lesions (Fig 14). These 

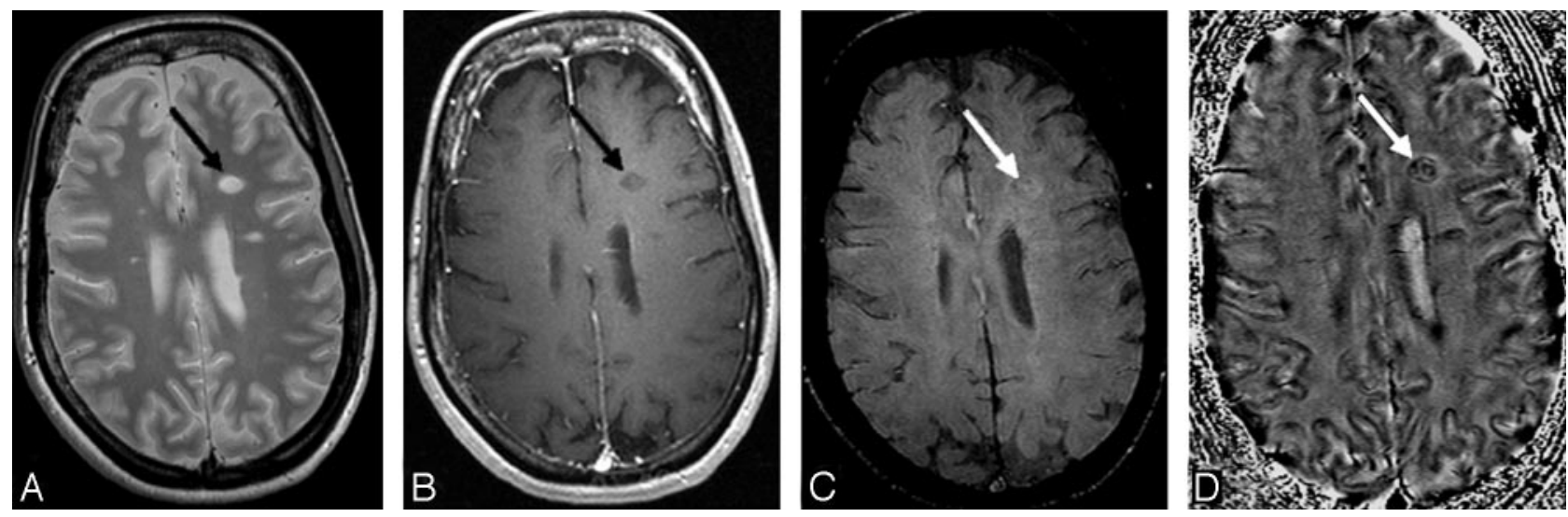

Fig 14. Another MS case shows a ringlike structure in the SWl filtered-phase image. The lesion of interest is highlighted by the either a white or black arrow. $A-D$, The $\mathrm{T} 2(A)$, $\mathrm{T} 1(B)$ original magnitude $(C)$, and SWI filtered-phase $(D)$ images show different lesion contrast. Iron appears in a ring structure with a smaller amount of iron scattered inside the lesion.

rings are often visible in SWI but not in conventional images. Longitudinal studies are needed to determine the role of SWI in measuring iron. In particular, SWI may help in determining the impact of iron deposition in MS.

\section{Vascular Malformations and Venous Disease}

Cerebral vascular malformations result from localized defects of vascular development. Most malformations are present at birth and can grow with time. In inherited forms, de novo lesions can also appear. ${ }^{52}$ Cerebral cavernous malformations (CCM), developmental venous angiomas (DVA), and capillary telangiectasias have slow flow and can be less conspicuous or entirely missed by conventional neuroimaging techniques, unlike high-flow AVMs. Although T2 ${ }^{\star}$ GE imaging can detect small venous structures, the incorporation of phase information in SWI offers improved sensitivity and can identify vascular structures that are often not visible on conventional imaging. ${ }^{53,54}$

CCMs. CCMs compose 10\%-20\% of all CNS vascular malformations and consist of abnormally enlarged capillary cavities surrounded by a single layer of endothelium without intervening brain parenchyma. ${ }^{55}$ CCMs have an estimated prevalence of $0.5 \%$ in the general population. They occur in both sporadic forms with a single lesion and in familial forms with multiple lesions. CCMs commonly present with seizures and recurrent cerebral hemorrhages. Because of the inherent risk of focal neurologic deficits secondary to repeated hemorrhages, precise detection of these angiographically occult lesions and screening of family members of patients with familial CCMs are important. The MR imaging findings of CCMs are variable, depending on the presence of hemorrhage and calcifications, but lesions typically appear as mixed-signal intensities, often described as "popcorn-like" in appearance, with a central reticulated core surrounded by a peripheral hypointense rim representing deposition of hemosiderin. Lesions that have previously bled are usually detectable on routine MR imaging due to the prominent signal intensity of hemorrhagic products. MR imaging with T2-weighted sequences has traditionally been the most accurate method to evaluate these lesions. ${ }^{56}$ However, if CCM lesions are intact and have not bled, they might not be detected or they may be seen only as a faintly enhancing parenchymal lesion.

SWI exploits different relaxation rates between venous and arterial blood as well as the phase changes caused by the susceptibility differences between oxygenated and deoxygenated hemoglobin. Therefore, this technique is exquisitely sensitive to these small differences and enhances the signal intensity-intensity loss in the venous circulation. ${ }^{2}$ As a result, the hemosiderin rim around CCMs appears clearly as a hypointense signal intensity on SWI images (Fig 15). Indeed, early studies have suggested that SWI was more accurate than $\mathrm{T} 2{ }^{\star} \mathrm{GE}$ imaging in detecting CCMs. ${ }^{54,57,58}$ Familial cavernomas are at especially high risk for hemorrhage and for developing new lesions with time. A study of 15 patients with familial CCMs reported that the sensitivity of SWI in identifying the number of CCM lesions was significantly higher than that of T2-weighted fast spin-echo (FSE) and GRE sequences. ${ }^{59}$ In summary, SWI depicts more small CCMs when compared with other conventional sequences. Also, because of its high degree of sensitivity, SWI can help confirm the diagnosis of CCM in patients presenting with cerebral hemorrhage. ${ }^{2}$

DVAs. DVAs are congenital anatomic variants of venous drainage and are the most common type $(\leq 60 \%)$ of all cerebral vascular malformations. ${ }^{60}$ Venous angiomas drain normal brain parenchyma and are often found incidentally. ${ }^{60}$ They consist of thickened hyalinized veins, which lack elastic tissue and are devoid of arterial-to-venous shunting. They coalesce into a large drainage vein and classically appear as a caput medusa. They are typically asymptomatic, do not have a high propensity to bleed, and generally do not require any neurosurgical intervention. ${ }^{61}$ However, DVAs are frequently associated with other vascular malformations, in particular cavernous angiomas, which are less innocuous, as discussed previously. Therefore, coincident vascular lesions need to be actively sought by appropriate additional imaging techniques. These types of low-flow malformations have been designated "occult" because they are usually not visible on conventional cerebral angiography, unlike high-flow AVMs. SWI can demonstrate conspicuously both numerous deep medullary veins and collector veins that are hardly visible on T2-weighted images or proton-attenuation images. SWI allows better visualization of DVAs without requiring contrast media, as shown in Fig 16.

Sturge-Weber Syndrome. Sturge-Weber syndrome (SWS) is a rare neurocutaneous disorder, typically manifested in children and characterized by a cutaneous angioma, glau- 

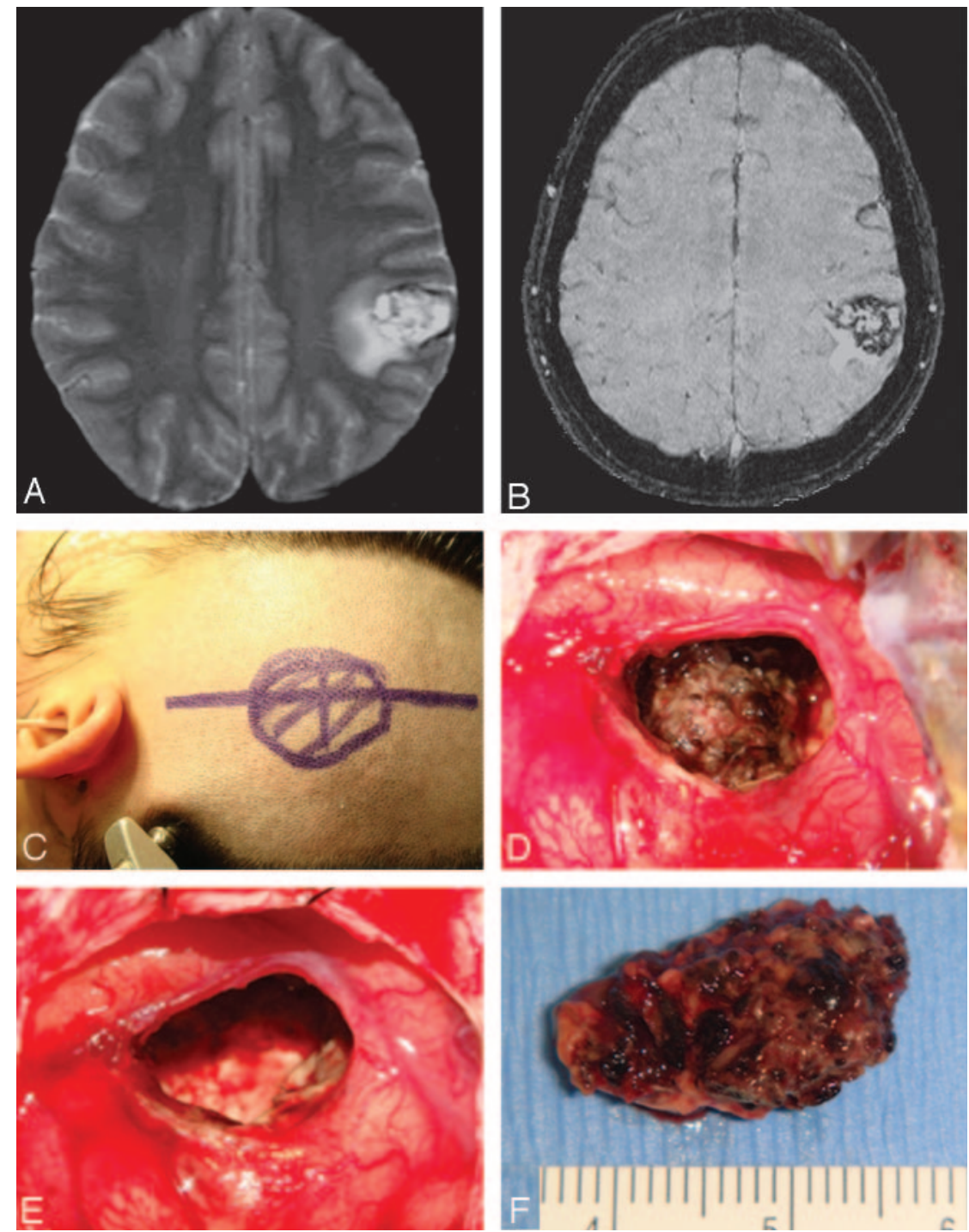

Fig 15. A 24-year-old Hispanic woman presenting with partial sensorimotor seizures. $A$, Axial T2-weighted FSE image shows a left parietal cavernous malformation. $B$, Because of its exquisite sensitivity in identifying venous structures and blood products, SWI provides a more complete evaluation of patients with CCM. $C-F$, Intraoperative photographs show circumferential dissection and complete resection of the left parietal cavernous malformation by using microsurgical techniques.
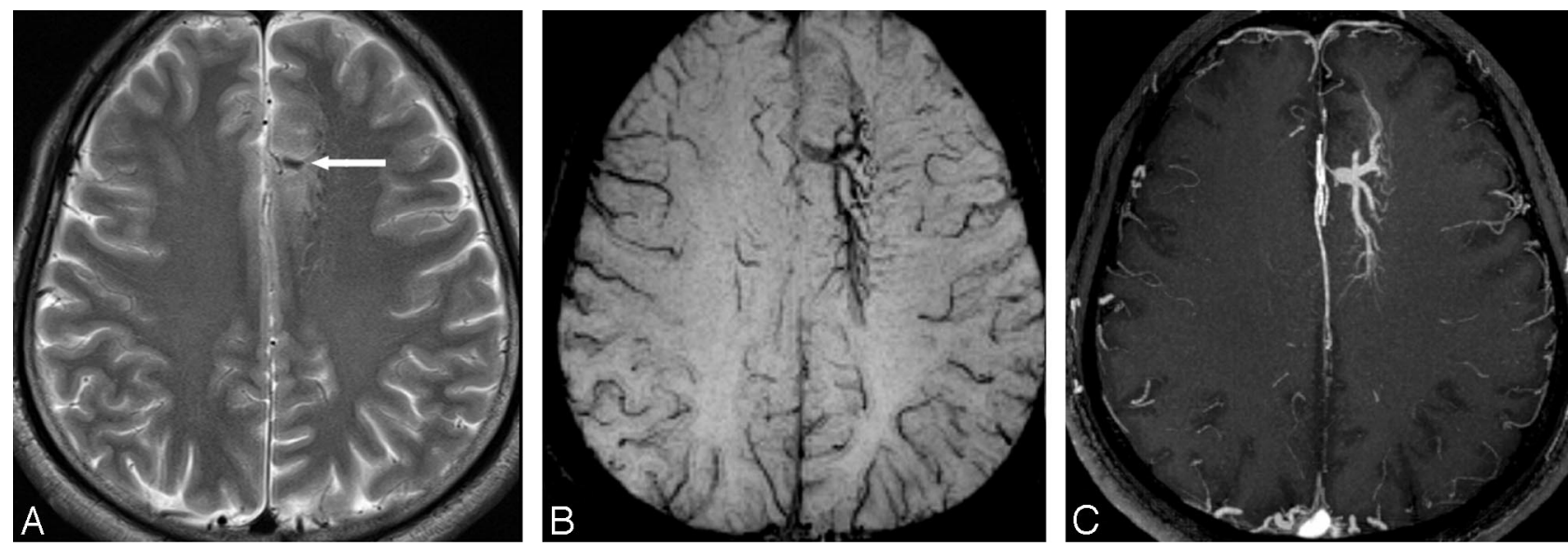

Fig 16. A 25-year-old man evaluated for long-term migraine problems. A, T2-weighted image shows a small vascular flow void (arrow) surrounded by an ill-defined region of mixed intensities in the left superior frontal gyrus. $B$, SWI depicts numerous fine well-delineated anomalous medullary veins that converge into a dilated transcortical collector vein. This forms the classic "caput medusa." C, Postcontrast 3D T1-weighted image reveals the dilated medullary veins and an enlarged collector vein in excellent agreement with SWI. 

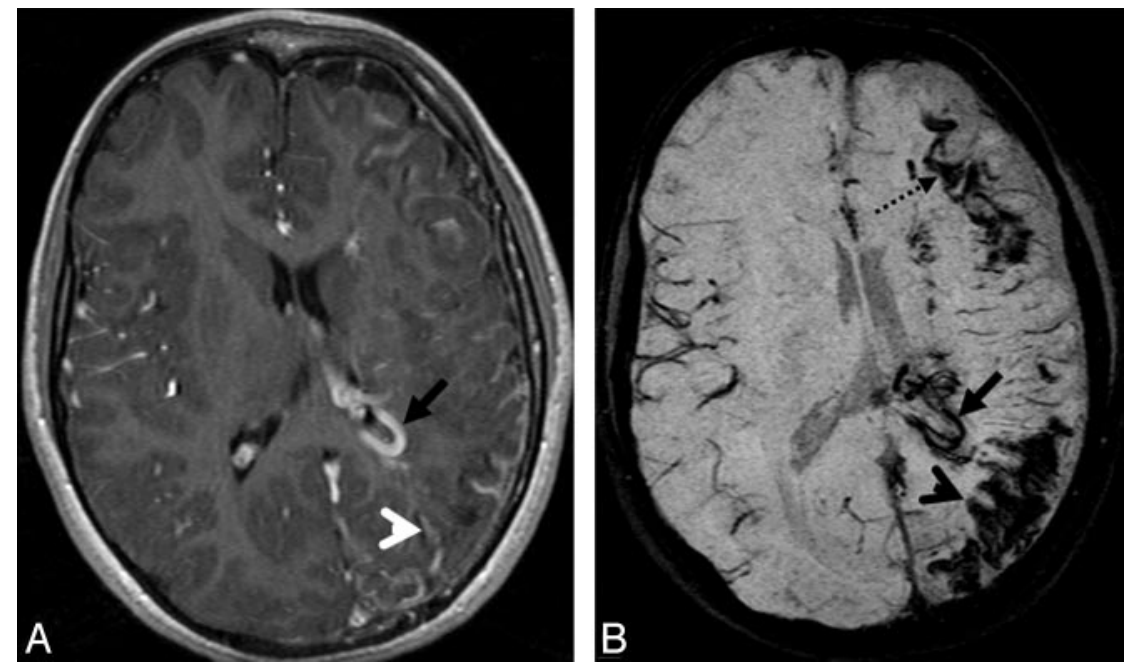

Fig 17. SWS in a 5-year-old girl. $A$, The postcontrast T1-weighted image shows enhancement of the leptomeninges (white arrowhead) and periventricular veins (arrow). $B$, SWI shows extensive hypointensity along the gyri (arrowhead and dotted arrow) due to calcification of the gyri. The abnormal periventricular veins (arrow) shows better detail than the postcontrast T1-weighted image $(A)$.

coma, and leptomeningeal venous angiomatosis. ${ }^{62,63}$ The anomalous venous plexus over the cerebral surface leads to abnormal cortical drainage, progressive venous stasis, and chronic hypoxia. The vascular malformation most commonly affects the parieto-occipital region but may also extend to the frontal or temporal lobes. Neurologic manifestations include seizures, hemiparesis, visual field deficits, and cognitive deficits. ${ }^{63}$ Calcification, impaired cortical venous outflow, and abnormal deep venous collaterals are common in SWS. Unilateral hemispheric involvement is most frequent. Children with unilateral SWS may show profound cognitive impairment, despite relatively limited unilateral cortical involvement. This observation suggests that conventional imaging may underestimate the extent of the structural and/or functional cerebral abnormalities contributing to cognitive decline in children with SWS. Moreover, the precise etiology and pathophysiology of this disease remain unknown, and the clinical course is often progressive and highly variable.

MR imaging has been useful for the diagnosis and evaluation of the severity of brain involvement in SWS. Specifically, T1-weighted gadolinium (Gd) postcontrast MR imaging can demonstrate focal cortical atrophy, contrast-enhancing leptomeningeal angiomatosis, and abnormal cerebral veins and is currently an imaging standard for clinical SWS diagnosis. ${ }^{63-65}$ In a recent study, we demonstrated the ability of SWI to detect both cortical abnormalities (including calcifications) and deep transmedullary veins in children with SWS. ${ }^{66}$ It remained unclear, however, to what extent SWI could complement conventional MR imaging (with contrast administration) in the clinical diagnosis and management of SWS. In a follow-up study, ${ }^{67}$ we hypothesized that SWI could provide better visualization of certain pathologic conditions in SWS than postcontrast T1 images. To evaluate this, we conducted a detailed qualitative and semiquantitative analysis and comparison of SWI and T1-Gd images of 12 prospectively studied children with SWS. ${ }^{67}$ Our results demonstrated that SWI indeed offered complementary information over conventional contrast-enhanced T1-weighted imaging. Specifically, SWI was superior to T1-Gd in characterizing abnormal trans- medullary veins, abnormal periventricular veins, cortical gyriform hypointensities, and gray/white matter junction abnormalities (Fig 17). Even without administration of any contrast agent, SWI revealed abnormal deep venous structures with a high contrast ratio and unparalleled detail, showing extensive abnormalities not reported by other imaging techniques. In contrast, T1-Gd was definitely better than SWI in identifying an enlarged choroid plexus and leptomeningeal angiomas.

In SWS, cortical abnormalities underlying the leptomeningeal angiomatosis can result from venous stasis leading to progressive hypoxia, necrosis, gliosis, and cortical calcification. Our data were consistent with previous imaging studies demonstrating the presence of abnormally enlarged veins in SWS, which are thought to provide collateral drainage for venous blood toward the deep cerebral venous system and can become increasingly prominent as surface veins occlude progressively. Our findings were also consistent with a recent SWS case report using SWI, which revealed abnormal transmedullary veins across the cerebral hemisphere in an infant, without the administration of contrast enhancement. ${ }^{68}$ In our study, SWI minimum intensity projection images clearly demonstrated that the abnormal transmedullary veins wind through the cerebral hemisphere and join the periventricular veins to form a drainage network for its neighboring cerebral territory. These enlarged regional transmedullary veins may have elevated deoxyhemoglobin concentration due to venous stasis and reduced perfusion.

In our recent multimodal imaging study by using fluorodeoxyglucose-positron-emission tomography and MR imaging, we demonstrated that hypometabolic cortical regions in SWS matched well with cortical susceptibility changes on SWI, whereas deep transmedullary veins typically drained from cortical areas directly adjacent to severely hypometabolic cortical regions. ${ }^{66}$ In addition, although T1-Gd images only showed morphologic changes of the veins, SWI offered not only morphologic but also physiologic information by characterizing the deoxygenation level of the venous blood. ${ }^{69}$ Although conventional MR imaging can delineate focal cortical atrophy and contrast-enhanced leptomeningeal angiomatosis, it is insensitive to calcifica- 

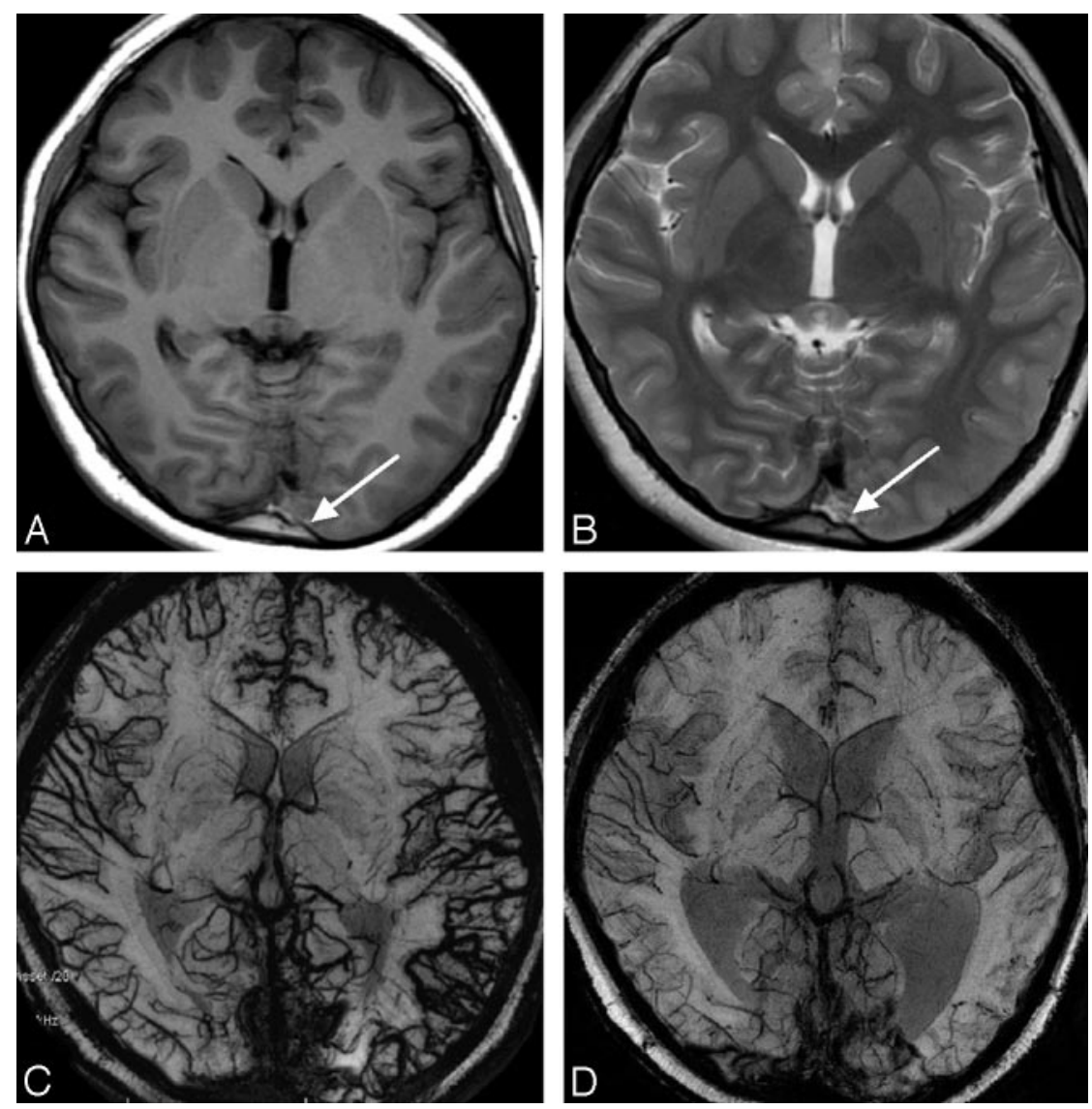

Fig 18. Cerebral venous sinus thrombosis. $A$ and $B$, Precontrast T1-weighted $(A)$ and T2-weighted $(B)$ images show normal cerebral parenchyma except in the sagittal sinus (arrow), where a hyperintense (T1) and isointense (T2) thrombus is replacing the normal low signal intensity of flowing blood. $C$, SWI shows significant engorgement of the venous system of the whole brain because of venous hypertension secondary to CVST. D, Three months later (after thrombolytic therapy), SWI shows a return to a normal-appearing venous system. Courtesy of Dr Guangbin Wang, Shandong Medical Imaging Research Institute, Jinan, China.

tions. Cortical pathology in SWS includes gliosis, atrophy, and perivascular calcifications, which can be detected as "tramtracklike" abnormalities on planar x-ray images and gyriform hyperattenuations on CT images. The location and pattern of the gyriform hypointense lesions in the cortex and the hypointensities at the junction of gray/white matter on SWI images are consistent with the pattern and location of CT findings of calcifications.

Altogether, SWI offers more information on venous abnormalities and calcifications, whereas T1-Gd demonstrates the characteristic findings of leptomeningeal angiomas and enlarged choroid plexus present in SWS. A complementary use of these 2 technologies, therefore, could reveal more information on SWS abnormalities than any of the 2 alone. Due to the progressive and highly variable nature of this complex neurocutaneous disorder, an accurate characterization of the extent and severity of brain involvement may improve patient management. The ability to show a redistribution of the venous system by SWI could assist in making decisions about therapeutic intervention.

SWI has been successfully applied in detecting cerebral vascular malformations, especially those with slow flow, including CCMs, DVAs, SWS, and capillary telangiectasias. ${ }^{1,53,54}$

Cerebral Venous Sinus Thrombosis. Cerebral venous sinus thrombosis (CVST) is an infrequent neurologic condition, which is notoriously difficult to diagnose because of its nonspecific clinical presentation. ${ }^{70}$ Direct evidence of sinus thrombosis such as the "triangle" sign and the "empty delta" on CT and loss of the normal flow voids on MR imaging can be easily missed unless clinical suspicion is high and direct signs are actively sought. ${ }^{71}$ Indirect signs of dural venous thrombosis are various, including cerebral edema, infarction, and hemorrhage. CVST can be potentially deadly if it remains undiagnosed and if thrombolytic treatment is not started in a timely manner. ${ }^{72,73}$ SWI has become a useful method to evaluate CVST by demonstrating venous stasis and collateral slow flow. Dural sinus thrombosis causes an increase of deoxyhemoglobin concentration in the involved veins. This appears as a prominent hypointense signal intensity on SWI.

In Fig 18, a 17-year-old adolescent boy had sinus venous thrombosis and accepted thrombolytic treatment. Prior to treatment, SWI showed engorgement of the venous system of the whole brain as a result of venous hypertension. Following intravenous thrombolysis, SWI demonstrated return to the baseline appearance of the normal venous system. If the radiologist had missed the direct signs of the thrombosis itself in T1- or T2-weighted imaging, the strikingly engorged veins would have led the radiologist to look for the cause. Venous hypertension can be detected at the early stage of CVST before infarction or hemorrhage occurs. Thus, SWI can assist in directing the radiologist to look for CVST while the patient is in the acute stage. 

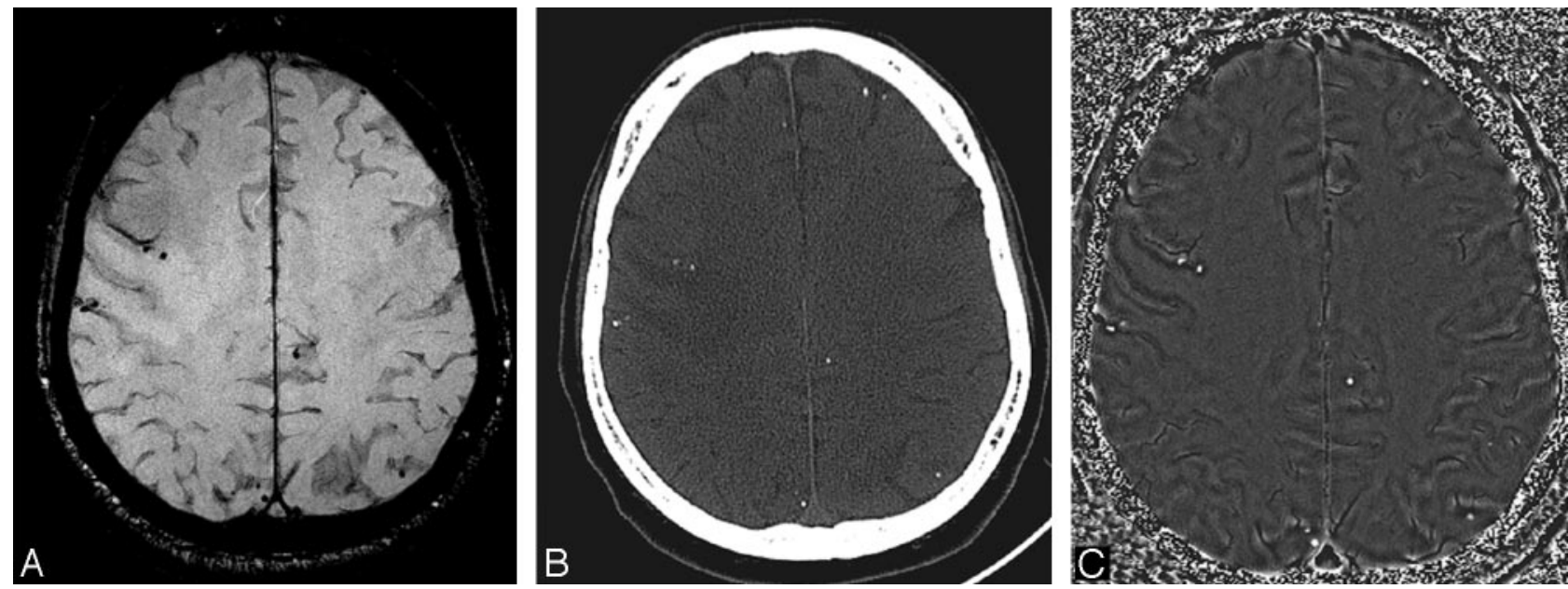

Fig 19. Conventional GE magnitude images show both calcifications and hemorrhage as areas of hypointense signal intensity. $A$, This is equally true for the SWI magnitude image with a TE of $40 \mathrm{~ms}$ at 1.5T. B, Usually, a CT scan is used to identify calcium. Here, the CT scan reveals many small calcifications. The SWI filtered-phase image is a means to differentiate paramagnetic from diamagnetic substances because the former will have a negative phase and the latter a positive phase (for a right-handed system). $C$, The phase image associated with the magnitude image in $A$ shows a 1-to-1 correspondence of the positive phase with the calcifications in the CT scan $(B)$.

\section{Neurocysticercosis}

Neurocysticercosis is the most common parasitic infection of the CNS worldwide. ${ }^{74}$ It is highly endemic in Central and South America, Mexico, Eastern Europe, Asia, Africa, and Spain. Tissue-invading larval forms of the pork tapeworm Taenia solium cause the disease. The ova of the tapeworm are spread via the fecal-oral route. The intermediate host is the pig, which harbors the larvae after eating ova, whereas humans are the definitive host. Infection occurs following ingestion of ova that develop into larvae (cysticerci) and lodge in soft tissues, such as skin, muscle, and the brain. In the CNS, T solium is deposited in the cerebral parenchyma, meninges, spinal cord, and eyes and forms small 1- to 2-mm fluid-filled cysticerci cysts. After the larvae die, the capsule thickens and the cysts degenerate. In the final stage, the small cystic lesions completely calcify. On CT, tiny calcified nodules without mass effect are typically noted. Figure 19 shows the usual multifocal small calcifications in the brain of a patient with a history of neurocysticercosis. Conventional GE and SWI magnitude images both show calcifications and hemorrhage as areas of hypointense signal intensity. In a recent article, we have demonstrated the value of SWI filtered-phase images as a means of differentiating paramagnetic from diamagnetic substances, because the former will have a negative phase and the latter, a positive phase (for a right-handed system) ${ }^{75}$ In a patient with a history of neurocysticercosis, the SWI phase image associated with the SWI magnitude image shows a 1-to-1 correspondence of the positive phase with the calcifications in the CT scan (Fig 19).

\section{Brain Tumors}

Recent advances in MR imaging have transformed imaging of brain tumors from a morphologic stage to a metabolic and functional level. Anatomic MR imaging has helped researchers and clinicians to diagnose, grade, and monitor therapeutic response with greater accuracy. MR spectroscopy, diffusion imaging, and perfusion studies have also made significant contributions in this respect.

Various imaging characteristics that have been suggested as predictors of glioma grade in humans include heterogeneity, contrast enhancement, mass effect, cyst formation or necrosis, metabolic activity, and CBV. In human glioma cells, the levels of ferritin and transferrin receptors detected at immunohistochemical analysis have been shown to correlate with tumor grade. Bagley et $\mathrm{al}^{76}$ found $\mathrm{T} 2^{\star} \mathrm{GE}$ MR images valuable in the preoperative grading of gliomas due to the increased susceptibility artifacts caused by hemorrhages. The development of SWI allows improved contrast and detection of both the venous vasculature and hemorrhage within tumors, which cannot be seen with conventional imaging methods. ${ }^{11,12,50,77-79}$ There is ample evidence that the growth of solid tumors, such as gliomas, is dependent on the angiogenesis of pathologic vessels. High-grade tumors such as glioblastomas often have a hemorrhagic component, which may be useful for staging (Fig 20). Christoforidis et al ${ }^{80}$ observed increased identification of microvascularity in glioblastoma with the help of high-resolution $\mathrm{T} 2{ }^{\star} \mathrm{GE}$ images at $8 \mathrm{~T}$. We have noted high rCBV values on perfusion imaging and high choline-creatine ratios on MR spectroscopy in tumors, which go hand in hand with evidence of blood products detected within the tumor matrix on SWI. However, larger comparative studies of perfusion imaging and SWI are needed to determine a more precise role of SWI in the grading of cerebral neoplasms.

Internal Vascular Architecture and Hemorrhage. With SWI, the internal architecture of the tumor is better defined compared with conventional MR images as well as contrastenhanced T1-weighted images (Fig 21). Hemorrhage can mimic intratumoral or peritumoral venous structures due to the similar paramagnetic susceptibility effect produced by them. However, hemorrhage can be distinguished from veins if SWI is used both before and after administration of a contrast agent. Blood vessels will change their signal intensity, whereas regions of tumoral hemorrhage appear unchanged.

We have found that SWI is equivalent to T1 contrastenhanced images in a majority of cases and has shown lesions better than $\mathrm{T} 1$ contrast-enhanced images in some cases. ${ }^{12} \mathrm{We}$ have also noted the increased sensitivity of noncontrast SWI in comparison with noncontrast spin-echo sequences. Contrast 

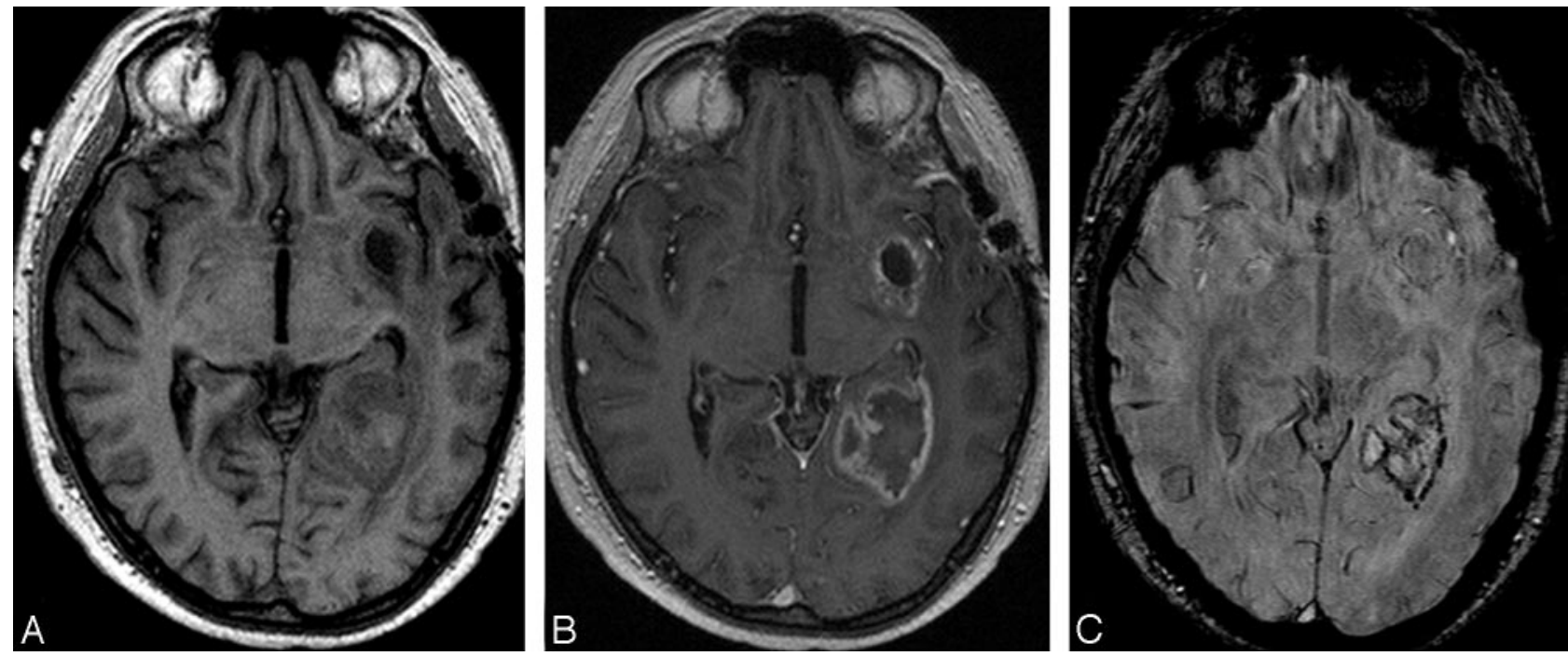

Fig 20. Left temporo-occipital high-grade glioma. SWI contrast-enhanced image $(C)$ shows similar boundary information and considerably more detail of the internal architecture of the tumor compared with the T1-weighted precontrast $(A)$ or postcontrast image $(B)$.
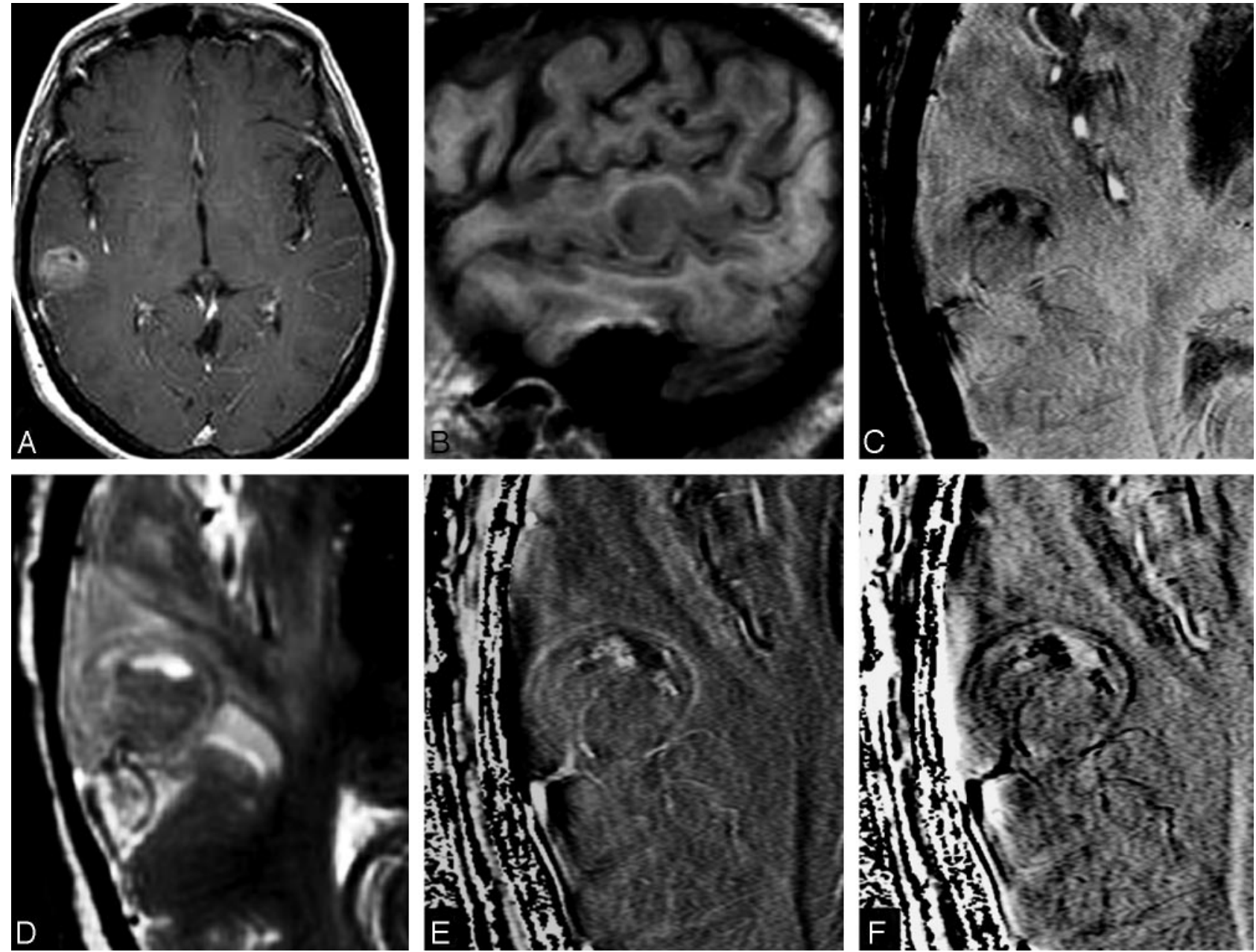

Fig 21. III-defined right temporal tumor histologically proved to be a glioblastoma. Compared with contrast-enhanced T1 $(A)$, precontrast T1 $(B)$, and FSE T2 $(D)$ images, SWI $(C)$ and phase images $(E$ and $F)$ are more sensitive in outlining areas of hemorrhage as well as the venous structures and thus can be a useful adjunct in defining the tumor characteristics. The phase images are shown for left-handed $(E)$ and right-handed systems $(F)$.

agent extravasation within the tumor does not normally lead to a major problem when SWI data are interpreted. However, if the contrast agent concentration is high enough, the phase may alias and the subsequent phase-mask processing may not lead to the expected signal-intensity enhancement.

The internal architecture of tumors varies significantly be- 

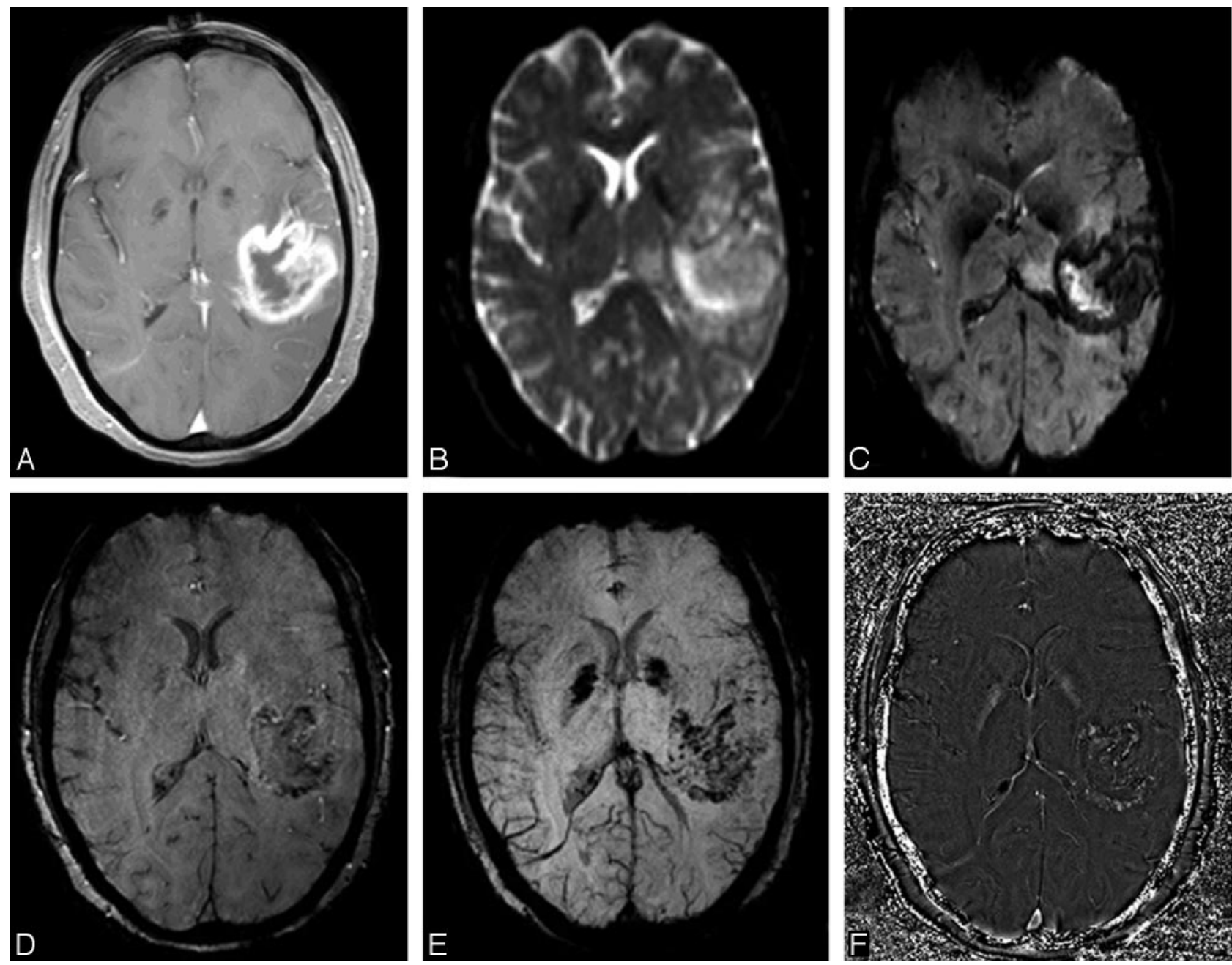

Fig 22. $A-C$, Left temporal glioblastoma. High-grade gliomas are well depicted on usual diagnostic sequences. $D-F$, However, SWI and phase images provide valuable additional information on the internal vascular architecture and presence of microhemorrhages within the tumor; $F$, phase image for a left-handed system.

tween SWI and contrast-enhanced T1 imaging. Figure 22 shows an example of the internal vascular structure of a lesion that is not visible even with the use of a contrast agent. Internal architecture on contrast-enhanced $\mathrm{T} 1$ is determined by the presence of necrosis, cysts, and tumor boundaries, whereas the internal architecture on SWI is determined mostly by blood products, either from spontaneous bleeding as commonly seen in high-grade tumors or from surgical trauma. This difference in image appearance can allow recurrent tumor to be distinguished from postsurgical changes. On SWI images, necrotic regions become high in signal intensity compared with the low-signal-intensity tumor parenchyma.

The growth of solid tumors, such as gliomas, is dependent on the angiogenesis of pathologic vessels. ${ }^{81,82}$ High-grade tumors often have a hemorrhagic component, which may be useful for staging. ${ }^{79}$ Postcontrast T1-weighted imaging is one of the most important sequences in evaluating brain tumors. However, extravasation of the contrast agent can often lead to diffuse enhancement of a tumor, thereby resulting in imprecise visualization of its inner structure and vascular components. It has been proved that SWI can delineate the inner structure and boundary of tumors and detect both the venous vasculature and hemorrhage within tumors. ${ }^{11,12,50,77-79}$ As shown in Fig 23, SWI clearly shows not only the boundary of the tumor but also different parts of the tumor and hemorrhage inside the tumor (the SWI phase image shows the thalamostriate veins compressed to the right by the tumor). Another case (Fig 24) is a meningioma located in the left posterior horn of the lateral ventricle. The tumor is diffusely enhanced in postcontrast T1-weighted imaging. SWI shows the draining vein of the meningioma very clearly. Meningiomas have very typical characteristics on conventional MR imaging and usually can be diagnosed easily. However, because meningiomas have an abundant blood supply, it is important to identify the location of the supplying arteries and draining veins when planning surgery. SWI can also be used to find a tumor indirectly by its high sensitivity to blood products. $^{11,77,83}$

Pre- and Postcontrast SWI. Hemorrhages can be easily distinguished from veins if SWI is performed both before and after administration of contrast agent. Blood vessels will change their signal intensity due to the contrast agent, but signal intensity from hemorrhage will not change. In Fig 25, a tumor is located in the posterior horn of the left lateral ventricle. Postcontrast SWI shows more hypointense signal intensity compared with SWI without a contrast agent, which indicates that these newly appearing hypointensities are vessels. 

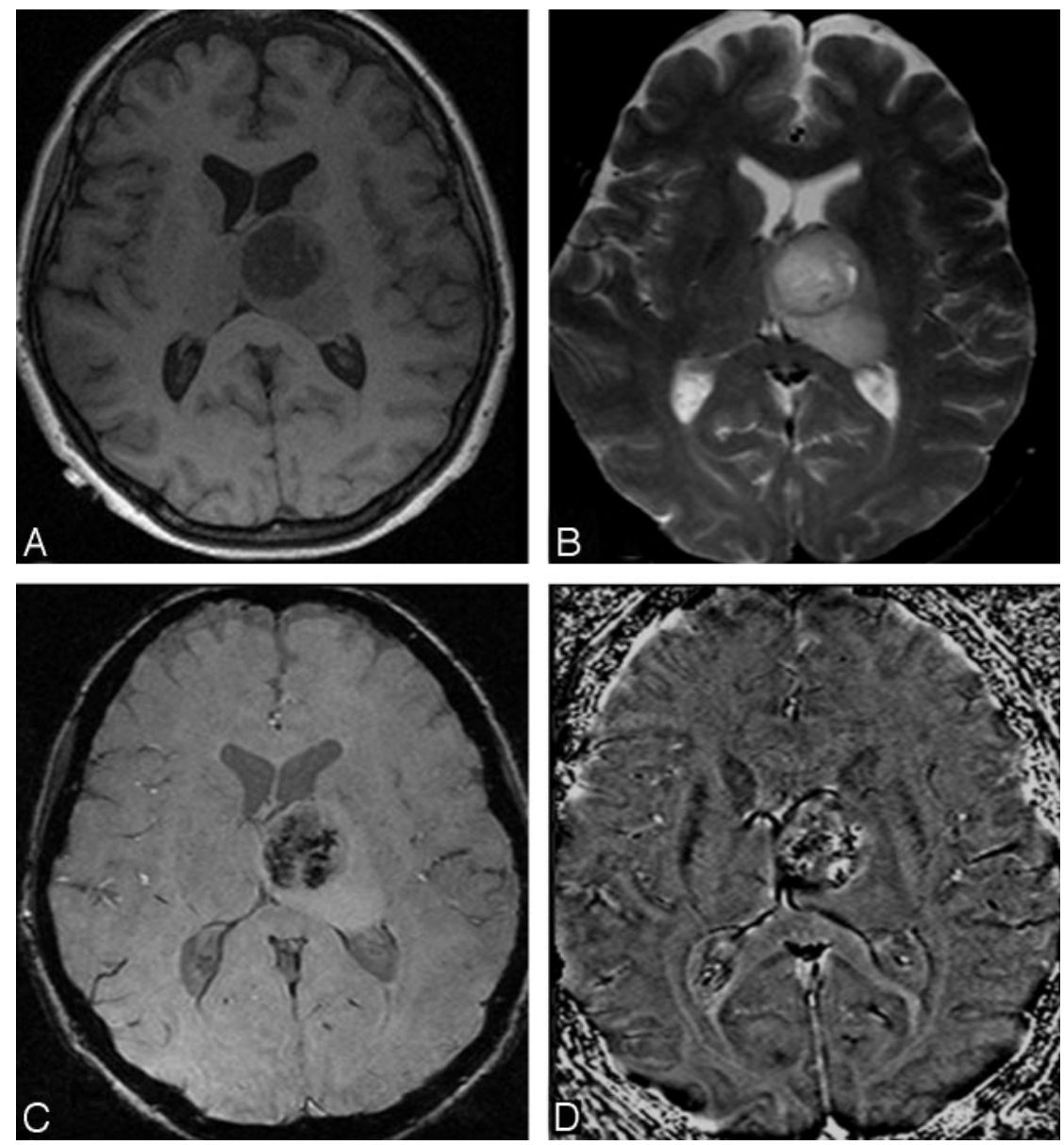

Fig 23. Left thalamic glioma. The size, location, and surrounding edema are well shown on the T1-weighted $(A)$ and T2-weighted images $(C)$. $B$, However, SWI delineates the interior structure and hemorrhages inside the tumors. $D$, The SWI phase image shows that the thalamostriate veins are compressed to the right by the tumor.
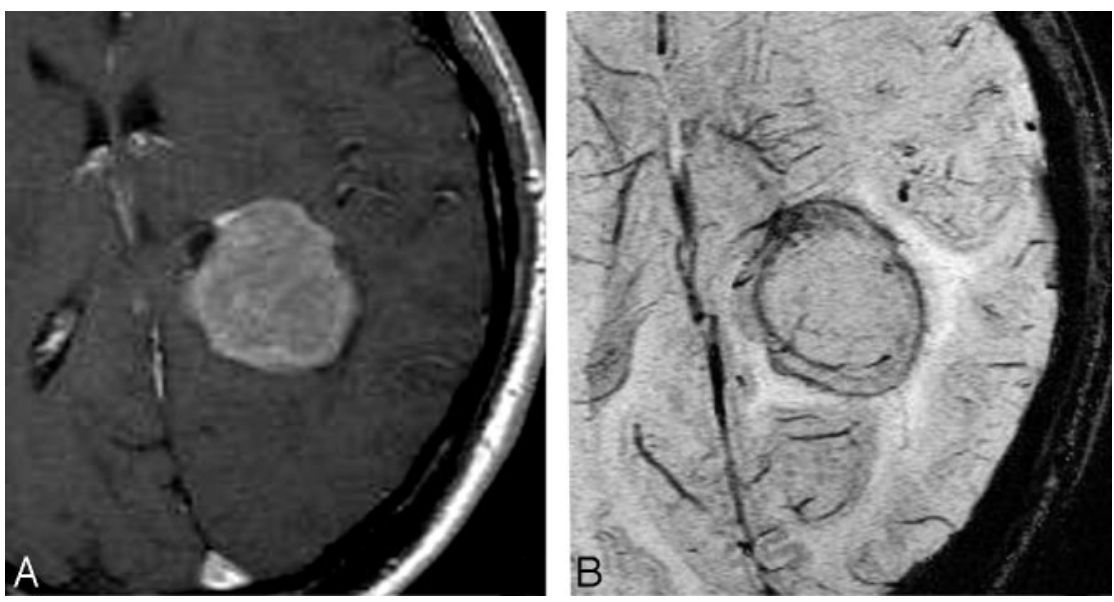

Fig 24. A meningioma located in the left posterior horn of the lateral ventricle. $A$, The tumor is diffusely enhanced in the postcontrast T1-weighted image. $B$, SWI delineates the veins around the tumor.

Intratumoral Calcification. Calcification is a very important indicator in diagnosis and differential diagnosis of brain tumors. Calcification cannot be definitively identified by MR imaging because its signals are variable on conventional spinecho T1- or T2- weighted images ${ }^{84-86}$ and cannot be differentiated from hemorrhage by GE images because both will cause local magnetic field changes and appear as hypointensities. However, as discussed in the neurocysticercosis case above, phase images can help differentiate calcification from hemorrhage because calcification is diamagnetic, whereas hemorrhage is paramagnetic, resulting in opposite signal intensities on SWI phase images. ${ }^{75}$ Figure 26 is a case of a histologically confirmed oligodendroglioma with intratumoral calcification identified by CT. The hypointensity shown on SWI magnitude images cannot be identified as either calcification or hemorrhage. On SWI filtered-phase images, the veins along the lat- 

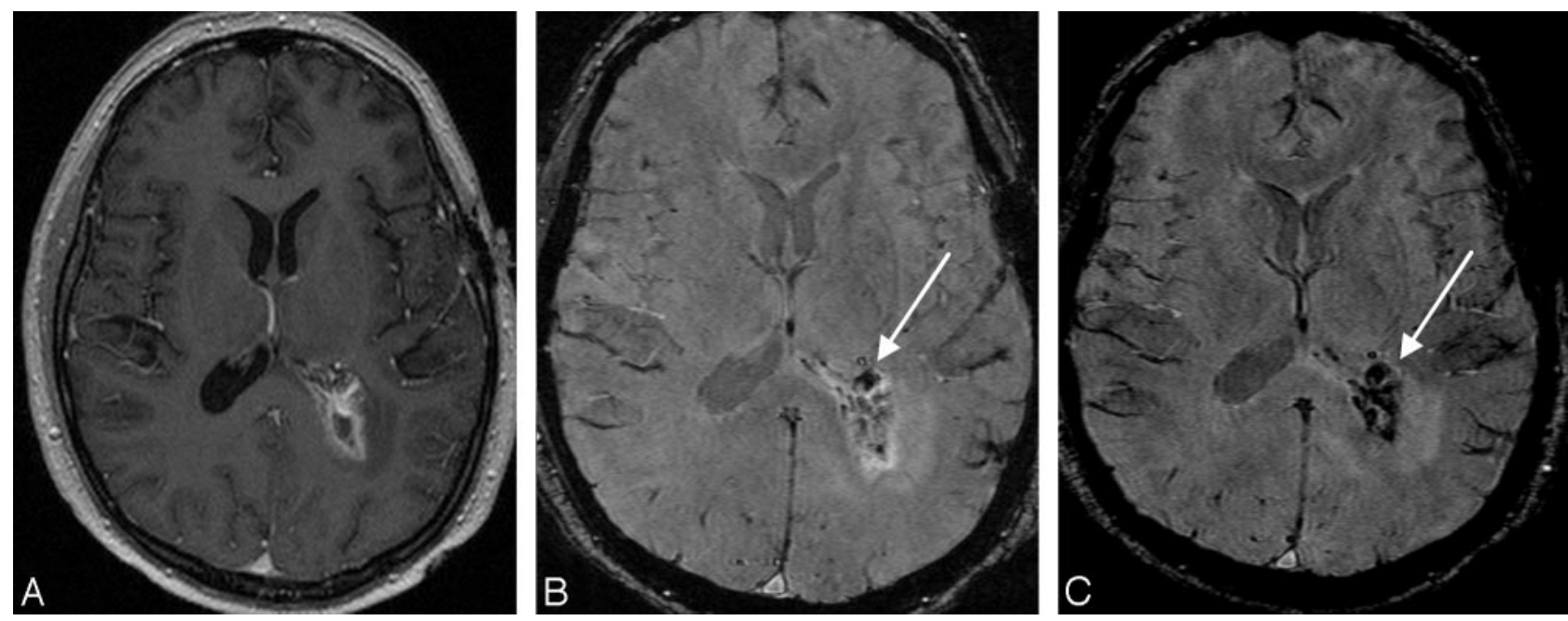

Fig 25. A tumor located in the posterior horn of the left lateral ventricle. A, Postcontrast T1-weighted image shows enhancement of the tumor periphery. $B$, Precontrast SWI shows a small hemorrhage inside the tumor (arrow). $C$, In postcontrast SWI, a new hypointense signal intensity appears after contrast agent injection, which indicates the presence of intratumoral vessels; the signal intensity from the hemorrhage inside the tumor remains unchanged (arrow).
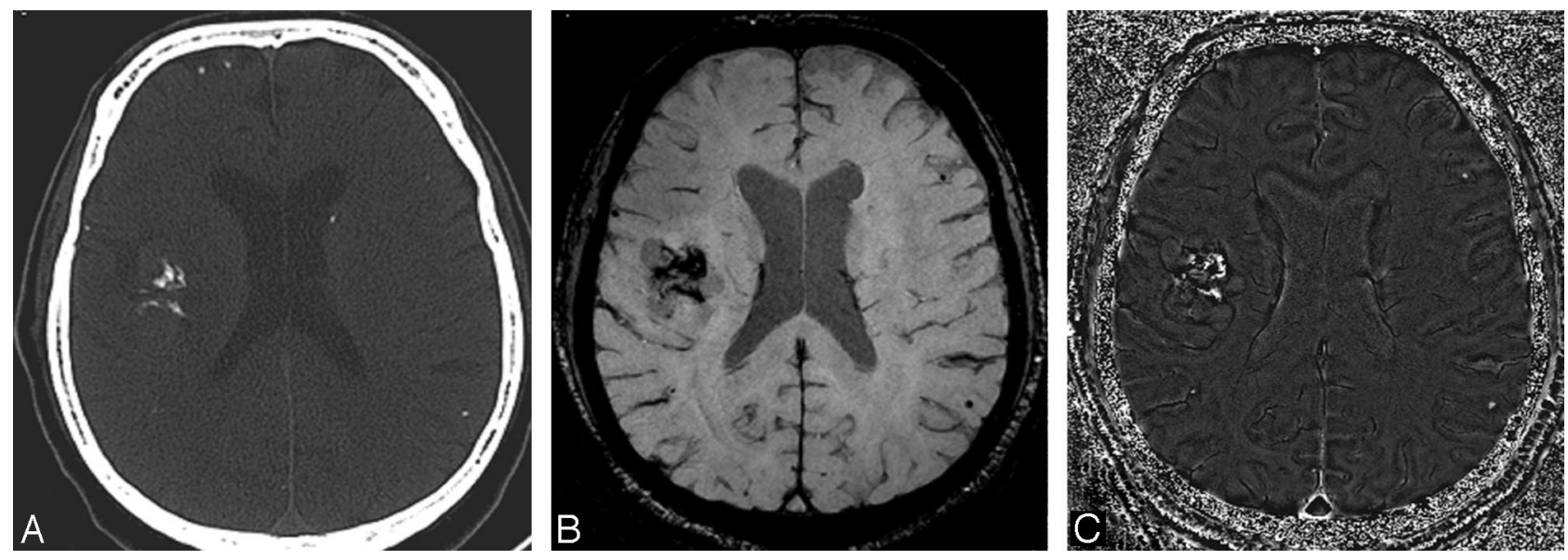

Fig 26. An oligodendroglioma in the right frontoinsular region. $A$, CT scan shows patchy calcification inside the tumor. $B$, SWI magnitude image shows hypointense signal intensity inside the tumor but cannot identify whether the hypointensity is hemorrhage or calcification. $C$, On the SWI phase image, calcification is identified and matches the CT results well.

eral ventricle and sulci appear dark, which means the hyperintensities inside the tumor are diamagnetic (ie, they are calcifications, rather than hemorrhage).

Radiation-Induced Changes. SWI can detect edema surrounding metastasis and hemorrhage within metastatic lesions. Many cerebral parenchymal metastatic lesions, including those from renal cell carcinoma, melanoma, and bronchogenic carcinoma, frequently show intratumoral hemorrhage. However, SWI cannot differentiate high-grade primary neoplasm and solitary hemorrhagic metastasis at present. SWI also appears to be useful in identifying the hemorrhagic changes that can be seen following radiation therapy. We recently imaged a patient with bilateral periventricular metastases from renal cell carcinoma who was treated with gamma knife radiosurgery 8 months previously (Fig 27). The postcontrast T1-weighted imaging failed to demarcate the site of the right periventricular tumor clearly. Presumably, this is because the blood-brain barrier remained intact; and because there was no contrast agent leaking from the vessels, the treated lesion did not enhance. ${ }^{87,88}$ In this case, SWI helped to detect the existence of postradiation changes by showing hemorrhaging at the treatment sites.

\section{Future Directions}

Although SWI interpretation will require some experience, increasing its clinical use will, no doubt, reveal new applications. To date, the strongest indications for SWI are in neurovascular and neurodegenerative disease applications. On the basis of additional information provided by SWI, we have defined a standard neuroprotocol, including conventional MR imaging, diffusion tensor imaging, PWI, and SWI. This protocol is being used by the MR Imaging Institute for Biomedical Research (Detroit, Mich) for the Neurovascular Imaging Center of Excellence (NICE) project. The major areas being considered for NICE are the following: aging, atherosclerosis, MS, occult vascular disease, stroke, trauma, and tumors. We invite all clinical centers that would like to participate in this worldwide data base effort to contact the corresponding author. We believe that the ever-expanding array of technology that can be used for neuroimaging may transform the field of radiology 

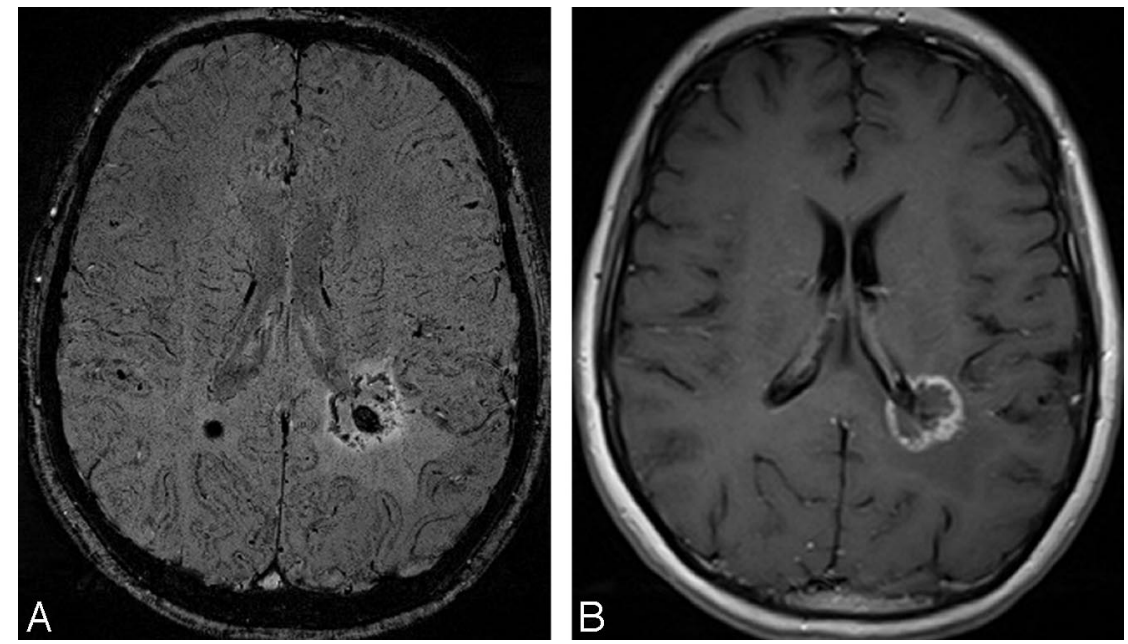

Fig 27. Bilateral periventricular metastases from renal cell carcinoma treated with gamma knife radiosurgery. SWI $(A)$ effectively identifies areas of microhemorrhages consistent with radiation necrosis, compared with the contrast-enhanced T1-weighted image $(B)$.

from simply being a diagnostic service to a means of studying the progression and etiology of disease.

\section{Acknowledgments}

We thank Alexander Boikov and Charbel Habib for proofing and other contributions to this article.

\section{References}

1. Haacke EM, Mittal S, Wu Z, et al. Susceptibility-weighted imaging: technical aspects and clinical applications, Part I. AJNR Am J Neuroradiol. 2009;30: 19-30

2. Tong KA, Ashwal S, Obenaus A, et al. Susceptibility-weighted MR imaging: a review of clinical applications in children. AJNR Am J Neuroradiol 2008;29:9-17

3. Brown AW, Elovic EP, Kothari S, et al. Congenital and acquired brain injury. Part 1. Epidemiology, pathophysiology, prognostication, innovative treatments, and prevention. Arch Phys Med Rehabil 2008;89:S3-8

4. Tong KA, Ashwal S, Holshouser BA, et al. Hemorrhagic shearing lesions in children and adolescents with posttraumatic diffuse axonal injury: improved detection and initial results. Radiology 2003;227:332-39

5. Tong KA, Ashwal S, Holshouser BA, et al. Diffuse axonal injury in children: clinical correlation with hemorrhagic lesions. Ann Neurol 2004;56:36-50

6. Babikian T, Freier MC, Tong KA, et al. Susceptibility weighted imaging: neuropsychologic outcome and pediatric head injury. Pediatr Neurol 2005; 33:184-94

7. Mannion RJ, Cross J, Bradley P, et al. Mechanism-based MRI classification of traumatic brainstem injury and its relationship to outcome. J Neurotrauma 2007;24:128-35

8. Ashwal S, Babikian T, Gardner-Nichols J, et al. Susceptibility-weighted imaging and proton magnetic resonance spectroscopy in assessment of outcome after pediatric traumatic brain injury. Arch Phys Med Rehabil 2006; 87:S50-58

9. Schellinger PD, Jansen O, Fiebach JB, et al. A standardized MRI stroke protocol: comparison with $\mathrm{CT}$ in hyperacute intracerebral hemorrhage. Stroke 1999;30:765-68

10. Hermier M, Nighoghossian N. Contribution of susceptibility-weighted imaging to acute stroke assessment. Stroke 2004;35:1989-94

11. Sehgal V, Delproposto Z, Haacke EM, et al. Clinical applications of neuroimaging with susceptibility-weighted imaging. J Magn Reson Imaging 2005;22: $439-50$

12. Thomas B, Somasundaram S, Thamburaj K, et al. Clinical applications of susceptibility weighted MR imaging of the brain: a pictorial review. Neuroradiology 2008;50:105-16

13. Wycliffe ND, Choe J, Holshouser B, et al. Reliability in detection of hemorrhage in acute stroke by a new three-dimensional gradient recalled echo susceptibility-weighted imaging technique compared to computed tomography: a retrospective study. J Magn Reson Imaging 2004;20:372-77

14. Schellinger PD, Thomalla G, Fiehler J, et al. MRI-based and CT-based thrombolytic therapy in acute stroke within and beyond established time windows: an analysis of 1210 patients. Stroke 2007;38:2640-45
15. Flacke S, Urbach $\mathrm{H}$, Block $\mathrm{W}$, et al. Perfusion and molecular diffusionweighted MR imaging of the brain: in vivo assessment of tissue alteration in cerebral ischemia. Amino Acids 2002;23:309-16

16. Chalela JA, Haymore JB, Ezzeddine MA, et al. The hypointense MCA sign. Neurology 2002;58:1470

17. Hermier M, Nighoghossian N, Derex L, et al. MRI of acute post-ischemic cerebral hemorrhage in stroke patients: diagnosis with $\mathrm{T} 2^{*}$-weighted gradientecho sequences. Neuroradiology 2001;43:809-15

18. Kidwell CS, Saver JL, Villablanca JP, et al. Magnetic resonance imaging detection of microbleeds before thrombolysis: an emerging application. Stroke 2002;33:95-98

19. Nighoghossian N, Hermier M, Adeleine $\mathrm{P}$, et al. Old microbleeds are a potential risk factor for cerebral bleeding after ischemic stroke: a gradient-echo $\mathrm{T} 2{ }^{*}$ weighted brain MRI study. Stroke 2002;33:735-42

20. Coutts S, Frayne R, Sevick R, et al. Microbleeding on MRI as a marker for hemorrhage after stroke thrombolysis. Stroke 2002;33:1457-58

21. Chalela JA, Kang DW, Warach S. Multiple cerebral microbleeds: MRI marker of a diffuse hemorrhage-prone state. J Neuroimaging 2004;14:54-57

22. Fazekas F, Kleinert R, Roob G, et al. Histopathologic analysis of loci of signal loss on gradient-echo $\mathrm{T} 2^{\star}$-weighted MR images in patients with spontaneous intracerebral hemorrhage: evidence of microangiopathy-related microbleeds. AJNR Am J Neuroradiol 1999;20:637-42

23. Von Kummer R. MRI: the new gold standard for detecting brain hemorrhages. Stroke 2002;33:1748-49

24. Akter M, Hirai T, Hiai Y, et al. Detection of hemorrhagic hypointense foci in the brain on susceptibility-weighted imaging clinical and phantom studies. Acad Radiol 2007;14:1011-19

25. Greer DM, KoroshetzWJ, Cullen S, et al. Magnetic resonance imaging improves detection of intracerebral hemorrhage over computed tomography after intra-arterial thrombolysis. Stroke 2004;35:491-95

26. Knopman DS. Cerebrovascular disease and dementia. Br J Radiol 2007;80:S121-27

27. Haacke EM, DelProposto ZS, Chaturvedi S, et al. Imaging cerebral amyloid angiopathy with susceptibility-weighted imaging. AJNR Am J Neuroradiol 2007;28:316-17

28. Greenberg SM, Eng JA, Ning M, et al. Hemorrhage burden predicts recurren intracerebral hemorrhage after lobar hemorrhage. Stroke 2004;35:1415-20

29. Vernooij MW, van der Lugt A, Ikram MA, et al. Prevalence and risk factors of cerebral microbleeds: the Rotterdam Scan Study. Neurology 2008;70:1208-14

30. Larsen JP, Britt W, Kido D, et al. Susceptibility weighted magnetic resonance imaging in evaluation of dementia. Radiology Case Reports 2007;2:102

31. Joutel A, Corpechot C, Ducros A, et al. Notch3 mutations in CADASIL, a hereditary adult-onset condition causing stroke and dementia. Nature 1996;383:707-10

32. Haacke EM, Cheng NY, House MJ, et al. Imaging iron stores in the brain using magnetic resonance imaging. Magn Reson Imaging 2005;23:1-25

33. Harder SL, Hopp KM, Ward H, et al. Mineralization of the deep gray matter with age: a retrospective review with susceptibility-weighted MR imaging. AJNR Am J Neuroradiol 2008;29:176-83

34. Jellinger KA. The role of iron in neurodegeneration: prospects for pharmacotherapy of Parkinson's disease. Drugs Aging 1999;14:115-40

35. Qian ZM, Wang Q. Expression of iron transport proteins and excessive iron 
accumulation of iron in the brain in neurodegenerative disorders. Brain Res Rev 1998;27:257-67

36. Swaiman KF. Hallervorden-Spatz and brain iron metabolism. Arch Neurol 1991;48:1285-93

37. Bakshi R, Shaikh ZA, Janardhan V. MRI T2 shortening (black T2) in multiple sclerosis: frequency, location, and clinical correlation. Neuroreport 2000;11: $15-21$

38. Ogg RJ, Langston JW, Haacke EM, et al. The correlation between phase shifts in gradient-echo MR images and regional brain iron concentration. Magn Reson Imaging 1999;17:1141-48

39. Haacke EM, Ayaz M, Khan A, et al. Establishing a baseline phase behavior in magnetic resonance imaging to determine normal vs. abnormal iron content in the brain. J Magn Reson Imaging 2007;26:256-64

40. Qian ZM, Shen X. Brain iron transport and neurodegeneration. Trends $\mathrm{Mol}$ Med 2001;7:103-08

41. Martin WR, Wieler M, Gee M. Midbrain iron content in early Parkinson disease: a potential biomarker of disease status. Neurology 2008;70:1411-17

42. Schenck JF, Zimmerman EA, Li Z. High-field magnetic resonance imaging of brain iron in Alzheimer disease. Top Magn Reson Imaging 2006;17:41-50

43. McNeill A, Birchall D, Hayflick SJ. T2* and FSE MRI distinguishes four subtypes of neurodegeneration with brain iron accumulation. Neurology 2008;70:1614-19

44. Schenck JF. Magnetic resonance imaging of brain iron. J Neurol Sci 2003; 207:99-102

45. Calabrese M, Filippi M, Rovaris M, et al. Morphology and evolution of cortical lesions in multiple sclerosis: a longitudinal MRI study. Neuroimage 2008;42:1324-28

46. Tan IL, van Schijndel RA, Pouwels PJ, et al. MR venography of multiple sclerosis. AJNR Am J Neuroradiol 2000;21:1039-42

47. Adams CW, Abdulla YH, Torres EM, et al. Periventricular lesions in multiple sclerosis: their perivenous origin and relationship to granular ependymitis. Neuropathol Appl Neurobiol 1987;13:141-52

48. Fog T. The topography of plaques in multiple sclerosis with special reference to cerebral plaques. Acta Neurol Scand Suppl 1965;15:1-161

49. Ludwin SK. Understanding multiple sclerosis: lessons from pathology. Ann Neurol 2000;47:691-93

50. Hammond KE, Lupo JM, Xu D, et al. Development of a robust method for generating 7.0 $\mathrm{T}$ multichannel phase images of the brain with application to normal volunteers and patients with neurological diseases. Neuroimage 2008;39:1682-92

51. Haacke EM, Makki M, Ge Y, et al. Characterizing iron deposition in multiple sclerosis lesions using susceptibility weighted imaging. J Magn Reson Imaging. 2009;29 In press

52. Brouillard P, Vikkula M. Genetic causes of vascular malformations. Hum Mol Genet 2007;16:140-49

53. Reichenbach JR, Jonetz-Mentzel L, Fitzek C, et al. High-resolution blood oxygen-level dependent MR venography (HRBV): a new technique. Neuroradiology 2001;43:364-69

54. Lee BC, Vo KD, Kido DK, et al. MR high-resolution blood oxygenation leveldependent venography of occult (low-flow) vascular lesions. AJNR Am J Neuroradiol 1999;20:1239-42

55. Battistini S, Rocchi R, Cerase A, et al. Clinical, magnetic resonance imaging, and genetic study of 5 Italian families with cerebral cavernous malformation. Arch Neurol 2007;64:843-48

56. Lehnhardt FG, von Smekal U, Ruckriem B, et al. Value of gradient-echo magnetic resonance imaging in the diagnosis of familial cerebral cavernous malformation. Arch Neurol 2005;62:653-58

57. Haacke EM, Xu Y, Cheng YC, et al. Susceptibility-weighted imaging (SWI). Magn Reson Med 2004;52:612-18

58. Rauscher A, Sedlacik J, Barth M, et al. Magnetic susceptibility-weighted MR phase imaging of the human brain. AJNR Am J Neuroradiol 2005;26:736-42

59. de Souza JM, Domingues RC, Cruz LC, et al. Susceptibility-weighted imaging for the evaluation of patients with familial cerebral cavernous malformations; a comparison with $\mathrm{T} 2$-weighted fast spin-echo and gradient-echo sequences. AJNR Am J Neuroradiol 2008;29:154-58

60. Abla A, Wait SD, Uschold T, et al. Developmental venous anomaly, cavernous malformation, and capillary telangiectasia: spectrum of a single disease. Acta Neurochir (Wien) 2008;150:487-89. Epub 2008 Mar 26

61. Topper R, Jurgens E, Reul J, et al. Clinical significance of intracranial developmental venous anomalies. J Neurol Neurosurg Psychiatry 1999;67:234-38
62. Comi AM. Update on Sturge-Weber syndrome: diagnosis, treatment, quantitative measures, and controversies. Lymphat Res Biol 2007;5:257-64

63. Lee JS, Asano E, Muzik O, et al. Sturge-Weber syndrome: correlation between clinical course and FDG PET findings. Neurology 2001;57:189-95

64. Fischbein NJ, Barkovich AJ, Wu Y, et al. Sturge-Weber syndrome with no leptomeningeal enhancement on MRI. Neuroradiology 1998;40:177-80

65. Reid DE, Maria BL, Drane WE, et al. Central nervous system perfusion and metabolism abnormalities in Sturge-Weber syndrome. J Child Neuro 1997; 12:218-22

66. Juhasz C, Haacke EM, Hu J, et al. Multimodality imaging of cortical and white matter abnormalities in Sturge-Weber syndrome. AJNR Am J Neuroradiol 2007;28:900-06

67. Hu J, Yu Y, Juhasz C, et al. MR susceptibility weighted imaging (SWI) complements conventional contrast enhanced T1 weighted MRI in characterizing brain abnormalities of Sturge-Weber syndrome. J Magn Reson Imaging 2008;28:300-07

68. Mentzel HJ, Dieckmann A, Fitzek C, et al. Early diagnosis of cerebral involvement in Sturge-Weber syndrome using high-resolution BOLD MR venography. Pediatr Radiol 2005;35:85-90

69. Shen $\mathrm{Y}$, Kou Z, Kreipke CW, et al. In vivo measurement of tissue damage, oxygen saturation changes and blood flow changes after experimental traumatic brain injury in rats using susceptibility weighted imaging. Magn Reson Imaging 2007;25:219-27

70. Ameri A, Bousser MG. Cerebral venous thrombosis. Neurol Clin 1992;10: 87-111

71. Tang PH, Chai J, Chan YH, et al. Superior sagittal sinus thrombosis: subtle signs on neuroimaging. Ann Acad Med Singapore 2008;37:397-401

72. Preter M, Tzourio C, Ameri A, et al. Long-term prognosis in cerebral venous thrombosis: follow-up of 77 patients. Stroke 1996;27:243-46

73. Hinman JM, Provenzale JM. Hypointense thrombus on T2-weighted MR imaging: a potential pitfall in the diagnosis of dural sinus thrombosis. Eur J Radiol 2002;41:147-52

74. Serpa JA, Yancey LS, White AC. Advances in the diagnosis and management of neurocysticercocis. Expert Rev Anti Infect Ther 2006;4:1051-61

75. Wu Z, Mittal S, Kish K, et al. Identification of calcification with magnetic resonance imaging using susceptibility-weighted imaging: a case study. J Magn Reson Imaging 2009;29:177-82

76. Bagley LJ, Grossman RI, Judy KD, et al. Gliomas: correlation of magnetic susceptibility artifact with histologic grade. Radiology 1997;202:511-16

77. Sehgal V, Delproposto Z, Haddar D, et al. Susceptibility-weighted imaging to visualize blood products and improve tumor contrast in the study of brain masses. J Magn Reson Imaging 2006;24:41-51

78. Noebauer-Huhmann IM, Pinker K, Barth M, et al. Contrast-enhanced, high resolution, susceptibility-weighted magnetic resonance imaging of the brain dose-dependent optimization at 3 tesla and 1.5 tesla in healthy volunteers. Invest Radiol 2006;41:249-55

79. Rauscher A, Sedlacik J, Fitzek C, et al. High resolution susceptibility weighted MR-imaging of brain tumors during the application of a gaseous agent. Rofo 2005;177:1065-69

80. Christoforidis GA, Kangarlu A, Abduljalil AM, et al. Susceptibility-based imaging of glioblastoma microvascularity at $8 \mathrm{~T}$ : correlation of MR imaging and postmortem pathology. AJNR Am J Neuroradiol 2004;25:756-60

81. Sharma S, Sharma MC, Gupta DK, et al. Angiogenic patterns and their quantitation in high grade astrocytic tumors. J Neurooncol 2006;79:19-30

82. Miller CR, Perry A. Glioblastoma. Arch Pathol Lab Med 2007;131:397-406

83. Haddar D, Haacke E, Sehgal V, et al. Susceptibility weighted imaging: theory and applications[in French]. J Radiol 2004;85:1901-08

84. Oot RF, New PF, Pile-Spellman J, et al. The detection of intracranial calcifications by MR. AJNR Am J Neuroradiol 1986;7:801-09

85. Avrahami E, Cohn DF, Feibel M, et al. MRI demonstration and CT correlation of the brain in patients with idiopathic intracerebral calcification. J Neurol 1994;241:381-84

86. Tsuchiya K, Makita K, Furui S, et al. MRI appearances of calcified regions within intracranial tumors. Neuroradiology 1993;35:341-44

87. Runge VM, Schoerner W, Niendorf HP, et al. Initial clinical evaluation of gadolinium DTPA for contrast-enhanced magnetic resonance imaging. Magn Reson Imaging 1985;3:27-35

88. Brant-Zawadzki M, Berry I, Osaki L, et al. Gd-DTPA in clinical MR of the brain: 1. Intraaxial lesions. AJR Am J Roentgenol 1986;147:1223-30 